\title{
ABSTRACTS
}

\section{SAEM-EMRS Combined Meeting, 18-20 October 1990, Edinburgh, Scotland.}

\section{A new parameter for predicting defibrillation success rate \\ C. G. BROWN, R. F. GRIFFITH, P. VAN PIGTEN, J. HOEKSTRA, G. NEJMAN, L. CASTO \& R. DYZWONCZYK}

Division of Emergency Medicine, Department of Preventive Medicine and Department of Anaesthesiology, The Ohio State University, Columbus, Ohio, U.S.A.

Study hypothesis. There are no guidelines to help determine the optimum time to defibrillate following the administration of an alpha-adrenergic agonist (AAA) during ventricular fibrillation (VF). Previous studies have shown that the median frequency (FM) of the VF ECG accurately estimates the metabolic state of the heart. We hypothesized that FM could predict the success of defibrillation and thus accurately determine the most appropriate time to defibrillate during VF.

Study population. Twenty-two mixed bread swine weighing $>15 \mathrm{Kgs}$ were studied.

Methods. VF was electrically induced and the VF ECG was analysed using fast Fourier analysis. After $10 \mathrm{~min}$ of VF, mechanical CPR was begun. After $3 \mathrm{~min}$ the animals received one of three AAA and CPR was continued. Defibrillation was attempted $31 / 2 \mathrm{~min}$ after drug administration. The average FM $20 \mathrm{~s}$ prior to defibrillation was calculated. The sensitivity of FM was determined.

Results. An FM of $\geq 7.72 \mathrm{~Hz}$ had a sensitivity of $100 \%$ in predicting successful defibrillation of VF.

Conclusion. The FM may serve as a valuable parameter to guide defibrillation therapy during VF. 


\title{
Prevention of cardiac arrest induced
} neurologic injury and delayed mortality by a high molecular weight chelator of iron

\author{
R. E. ROSENTHAL, G. H. MARSHALL Jr. \& R. F. \\ SHESSER
}

Department of Emergency Medicine, The George Washington University Medical Center, Washington, D.C., U.S.A.

Study hypothesis. Reactive iron has been implicated as an initiator of peroxidative neuronal damage following resuscitation from cardiac arrest (CA). Beneficial effects of post-ischemic iron chelation with deferoxamine (DFO) may be diminished bo the hypotensive effects of IV DFO. This study tests the hypothesis that a new hig molecular weight iron chelator (HMWC) without hypotensive effects will improve neurologic outcome and survival after CA.

Methods. In a non-randomized controlled trial, male Wistar rats, ventilated on room air (RA) underwent $6.5 \mathrm{~min} C A$ induced by intracardiac injection of $0.4 \mathrm{ml}$ cold $1 \% \mathrm{KCl}$ and thoracic compression. ROSC was accomplished with LACCPR synchronous with $100 \% \mathrm{O}_{2}$ ventilation. Fifty-six Survivors alternately received IV injections of either HMWC (DFO covalently attached to hydroxyethyl starch, Biomedical Frontiers, Inc.) at a dose of $100 \mathrm{mg} D F O / \mathrm{kg}$, or saline. Ventilation with $100 \% \mathrm{O}_{2}$ was continued for $30 \mathrm{~min}$ before return to RA. Animal survival was recorded daily. Neurologic deficit scores (NDS) of survivors were measured at days 1,3 , and 10 by blinded observers.

Results. NDS were significantly less (Wilcoxon Rank Sum; $\mathrm{P}<0.5 \mathrm{sig}$.) in the treated group at day $1(12.0 \pm 13.4 \mathrm{HMWC} ; 29.1 \pm 16.9$ Control; $\mathrm{P}=.0006)$ and day $3(5.3 \pm 7.1 \mathrm{HMWC} ; 19.0 \pm 14.7 \mathrm{Control} ; \mathrm{P}=.002)$ and approached significance at 9 day $10(2 \cdot 8 \pm 8 \cdot 7$ HMWC; $6.9 \pm 14.0$ Control; $P=.059)$. While mortality was similar (Pearson's chi-squared analysis; $\mathrm{P}<0.5 \mathrm{sig}$ ) during the first $24 \mathrm{~h}(5 / 28 \mathrm{HMWC}$, $4 / 28$ Control; $P=.72$ ), delayed mortality (days 1 through 10 ) among $24 \mathrm{~h}$ survivors was significantly reduced in the treated group $(13 \%)$ when compared to controls (42\%); $\mathrm{P}=.028$.

Conclusions. The covalent attachment of DFO to hydroxyethyl starch permitted safe administration of high dose DFO after CA. There was significant improvement $\stackrel{\bullet}{=}$ in neurologic outcome in those animals receiving HMWC following CA, thus $\stackrel{\oplus}{?}$ contributing to decreased delayed mortality with HMWC administration. 


\title{
Do CKMB results affect chest pain decision making in the ED?
}

\author{
G. P. YOUNG, J. R. HEDGES, W. B. GIBLER, T. R. \\ GREEN \& R. J. SWANSON \\ Portland Veteran Affairs Medical Center, Oregon Health Sciences University and \\ University of Cincinnati Medical Center
}

Study hypothesis. CKMB values will have a positive impact upon medical decision making in the evaluation of ED patients with chest pain (CP).

Study population. Patients were over 30 years old with $\mathrm{CP}$ and nondiagnostic ECGs. Patients were excluded if $\mathrm{CP}$ was explained by trauma or X-ray findings, if two surveys were not completed, or if followup data was inadequate.

Methods. Physicians completed entry and disposition surveys. CKMB levels were drawn every hour for up to $3 \mathrm{~h}$ (1-4 CKMBs). Physicians could admit their patients to the hospital at any time. Both surveys documented the physician estimate of MI probability, need for hospitalization and CCU admission. The second survey documented the relative importance of the CKMBs. The absolute log likelihood ratio (LLR) test measured the contribution of CKMB to decision certainty. The LLR is the absolute logarithm of the ratio of the post-test to pre-test odds. The LLR for different groups was compared using that test $(P<0.05)$.

Results. Of the 334 study patients 28 had MI. CKMB results were considered important for decision making by the physicians in $10 \mathrm{MI}$ patients and 101 of 306 nonMI patients. For MI patients, CKMBs affected the decision to admit to telemetry as opposed to CCU, but not the certainty of MI or the need for admission.

Conclusions. The rapid availability of serial CKMB results appears to affect decision making in one-third of ED patients with $\mathrm{CP}$ and nondiagnostic ECGs. For these patients, CKMB results raise the suspicion for MI in patients with MI and reduce the perceived need for CCU admission in patients without MI. 


\section{Effect of monomethoxypolyethyleneglycol- super oxide dismutase (peg-sod) on spinal cord ischemia in rabbit model}

K. N. HALL, W. G. BARSAM, R. V. W. DIMLICH, M. S. MILLER \& A. ERZIN

Department of Emergency Medicine, University of Cinncinnati Medical Center and Sterling Drug, Inc. Rensselaer, NY 12144, U.S.A.

Hypothesis. The use of PEG-SOD will produce a $50 \%$ improvement in neurologi包 outcome, mortality, and activity level after spinal cord ischemia in the rabbit.

Population. Forty-two New Zealand white male rabbits weighing $2.5-3.5 \mathrm{~kg}$.

Methods. The animals were subjected to spinal cord ischemia by clamping aorta distal to the left renal artery for $26 \mathrm{~min}$. The rabbits were randomly assigned to three groups: Group I - placebo $(n=16)$; Group II - 10000 U/kg IV PEG-S 9 P immediately prior to reperfusion $(n=13)$; Group III $-10000 \mathrm{U} / \mathrm{kg}$ IV PEG-SOL $20 \mathrm{~min}$ after reperfusion $(n=13)$. The animals were evaluated at one, two and sever: days by a blinded examiner. The subjects were classified by activity level (active $v s$ inactive including dead) and presence of hindlimb paralysis (not paralyzed $v \overrightarrow{\widehat{E}}$ paralyzed including dead). Activity level was defined as the animal's level of alertness and willingness to move. Death alone was also analysed as an outcome variable. The chi square and student's t-test were used as appropriate.

Results. Rabbits treated with PEG-SOD prior to reperfusion were significantl more active at all evaluation periods than those treated $20 \mathrm{~min}$ after reperfusion, of the control group $\left(P<0.05, X^{2}=6 \cdot 10\right)$. No significant difference in neurologiG outcome was found. A trend toward more deaths in the control group was found

\begin{tabular}{lcccc}
\cline { 2 - 5 } & Group & Active & Inactive & Dead \\
\cline { 2 - 5 } Activity & I $(\mathrm{n}=16)$ & 5 & 6 & 5 \\
level at & II $(\mathrm{n}=13)$ & 10 & 1 & 2 \\
$24 \mathrm{~h}$ & III $(\mathrm{n}=13)$ & 6 & 5 & 2 \\
\hline
\end{tabular}

Conclusions. PEG-SOD had a beneficial effect on activity level after spinal cor ischemia when given prior to reperfusion. Further study on a larger population is required to evaluate the full effect of PEG-SOD on neurologic outcome. 


\section{Outcome predictors of acetaminophen toxicity}

J. W. DONOVAN, M. J. GORAYEB, K. W. KULIG \& B. H. RUMACK

Capital Area Poison Center, Penn State University and Rocky Mountain Posion and Drug Center

Acetaminophen (APAP) levels are used to determine the need for $\mathrm{N}$-acetyl-cysteine (NAC) therapy, but degree of elevation and treatment times (T) beyond $8 \mathrm{~h}$ postingestion do not consistently correlate with the development of hepatotoxicity. This study was undertaken to test the validity of previously suggested parameters to predict outcome of those treated with NAC and their need for continued hospitalization. Initial APAP level, prothrombin time (PT), aminotransferases (AT), bilirubin (B), T, APAP half life ( $\left.\mathrm{T}^{1} / 2\right)$, and peak AT, B, and PT were recorded on all patients treated at one of the centres participating in a national study of a $49 \mathrm{~h}$ intravenous NAC protocol for APAP overdose. Negative outcome was defined as a peak AT $>1000 \mathrm{IU} / \mathrm{L}$. Predictive values (PV) of positive and negative tests were calculated, utilizing Bayesian probability analysis. Preliminary data in this group of patients presenting at 4-24h post-ingestion showed that no patients with $\mathrm{T}<8 \mathrm{~h}$ and only $1 / 41(2.4 \%)$ with initial AT $<2$ times normal developed toxicity (negative test PV $100 \%$ and $98 \%$ respectively). No patients meeting these criteria required additional therapy once NAC was completed. Other outcome predictors studied were not as consistently associated with outcome. 


\title{
Protective effects of Felodipine, Nimodipine and Verapamil against imiparmine-induced
} lethal cardiac conduction disturbances in the anaesthetized rat

\author{
D. F. SCHOORS, H. REYNAERT, H. SPAPEM, \\ L. HUYGHENS, W. VINCKEN \& L. CORNE
}

Department of Emergency and Intensive Care, University Hospital (VUB), Laarbeeklaam 101, B-109), Brussels, Belgium

Toxic concentrations of tricyclic antidepressant drugs (TCA), following overdose are frequently associated with seizures and both electrocardiographic and haemoe dynamic changes. We assessed the effect of verapamil $(\mathrm{V})$, felodipine $(\mathrm{F})$ ant nimodipine $(\mathrm{N})$ on imipramine (IMI) induced cardiac toxicity. In preliminary experiments minimal lethal dose of IMI was $10 \mathrm{mg} / \mathrm{kg}$ i.v. Forty-two male WistarKyoto rats, weighing $352 \pm 32 \mathrm{~g}$, anaesthetized with sodium pentobarbital $(40 \mathrm{mg} / \mathrm{kg}$ i.p.) were equipped with an intra-arterial catheter, allowing continuous recording of blood pressure (BP). ECG recording was performed using three subcutaneous needle electrodes. Both jugular veins were cannulated. The right jugular vein was used for perfusion of saline $(\mathrm{n}=7), \mathrm{V}(30$ and $100 \mu \mathrm{g} / \mathrm{kg} / \mathrm{min}$; $\mathrm{n}=14)$, $F$ ( 3 and $6 \mu \mathrm{g} / \mathrm{kg} / \mathrm{min} ; \mathrm{n}=14)$ or $\mathrm{N}(1 \mu \mathrm{g} / \mathrm{kg} / \mathrm{min} ; \mathrm{n}=7)$, in doses not significantly changing BP.

Fifteen minutes after starting the infusion a $10 \mathrm{mg} / \mathrm{kg}$ bolus injection of IMI was given through the left jugular vein. All rats pretreated with saline died within $2.33 \pm 0.75 \mathrm{~min}$. ECG recording showed sinus bradycardia, followed by atrio- and intraventricular conduction disturbances, with marked widening of QRS (from $0.037 \pm 0.003 \mathrm{~s}$ to $0.088 \pm 0.010 \mathrm{~s} ; \mathrm{P}<0.001)$ and significant increase of the RS height $(+53.1 \pm 11.1 \% ; \mathrm{P}<0.01)$. In contrast, rats pretreated with $\mathrm{V}$ (30 and $100 \mu \mathrm{g} / \mathrm{kg} / \mathrm{min}), F(3 \mu \mathrm{g} / \mathrm{kg} / \mathrm{min})$ or $\mathrm{N}(1 \mu \mathrm{g} / \mathrm{kg} / \mathrm{min})$ survived throughout the experiment, despite an initial fall in $\mathrm{BP}$, which was less pronounced then after saline. However, ECG monitoring showed still significant QRS widening and RS height increse. All rats, pretreated with $6 \mu \mathrm{g} / \mathrm{kg} / \mathrm{min}$ of $\mathrm{F}$, survived and IMI did not induce ECG changes (QRS width: $0.037 \pm 0.003 \mathrm{~s}$ before IMI $v$ s. $0.038 \pm 0.004 \mathrm{~s}$ after IMI; ns) (RS height: $+9.1 \pm 3.3 \%$; ns). These findings suggest that pretreatment with $\mathrm{V}, \mathrm{F}$ and $\mathrm{N}$ protects against the cardiac effects of IMI, possibly via preventing 
the catecholaminergic action. As far as felodipine is concerned, an additional interaction with the calmodulin- $\mathrm{Ca}^{2+}$ complex could play a role. A randomized, double-blind, placebo-controlled clinical trial is currently under investigation in our emergency department.

\section{Does paramedic-hospital base radio contact result in actions that deviate from standard treatment protocols?}

J. R. HOFFMAN, J. S. LUO \& D. L. SCHRIGER UCLA Emergency Medicine Center and UCLA School of Medicine, California, U.S.A.

Hypothesis. Radio contact between paramedics and base-hospital personnel rarely results in therapies not predicted by standard pre-hospital protocols.

Design. Retrospective survey of written and audio records for pre-hospital patients with a chief complaint of seizure, syncope, abdominal pain or altered mental status (AMS).

Study population. Six hundred and fifty-nine consecutive pre-hospital patients with one of the 4 listed chief complaints handled by a university hospital basestation over a one-year period $(23.4 \%$ of all runs). Patients with a history of primary trauma were excluded.

Methods. We reviewed all pre-hospital records, and scored each patient's care for use of therapies listed in standard treatment protocols for these complaints (e.g. IV access, oxygen, glucose and naloxone), as well as therapies not routinely included in such protocols (e.g. nitroglycerin or dopamine, endotracheal intubation). All charts containing unanticipated therapies were evaluated as to whether simple clinical findings could identify for paramedics 'special cases' in need of such action. Los Angeles County statistics were analysed to assess the cost-impact of limiting radio contact between paramedics and base-hospitals.

Results. Standard modalities were used in the large majority of patients: (IV in $94 \%$ and oxygen in $91 \%$ of all cases, glucose and naloxone in $87 \%$ of AMS cases). Fifteen unanticipated therapies were used in $13(2.0 \%)$ patients; in each case this was prompted by abnormal vital signs, respiratory distress, profuse diaphoresis, or a second prominent complaint.

Conclusions. Almost all patients with these chief complaints could be managed by paramedics using protocols in lieu of base-hospital contact. The rare patient who might benefit from radio contact can be identified by obvious clinical characteristics. Projected annual savings resulting from a $90 \%$ decrease in the number of radio contacts in Los Angeles are in the range of 2 to 3 million dollars. 


\section{The usefulness of a rapid CPK-MB assay in} the evaluation of emergency department patients with possible myocardial infarction $\stackrel{\mathscr{\leftrightarrow}}{\circ}$

G. B. GREEN, K. N. HANSEN, D. FLEETWOOD, D. W. CHAN, A. D. GUERCI \& G. D. KELEN

Division of Emergency Medicine, Division of Laboratory Medicine and Division of Cardiology, The Johns Hopkins University, Baltimore, MD, U.S.A.

Objectives. To determine sensitivity, specificity, of a new rapid CPK-MB assay and assess its potential utility for the ED.

Design. Descriptive study with 271 consecutively enrolled ED patients during 4-month period in 1989. Delayed identity-unlinked serologic analysis. Results available for clinical management.

Setting. Inner-city teaching hospital ED.

Patients. All ED patients with excess sera meeting criteria consisting of 9 strictl defined presentations consistent with possible myocardial ischemia.

Measurements. Sera was measured for CPK-MB using rapid Hybritech ICON $\mathrm{QSR}^{\mathrm{R}}$ assay and standard Hybritech Tandem-E $\mathrm{II}^{\mathrm{R}}$ assay.

Results. Rapid assay was easy to perform with results available in 10 minutesCompared to standard, the rapid assay was $100 \%$ sensitive and $96 \%$ specific, witto PPV of $75.0 \%$ and NPV of $100 \%$. There were 32 patients $(11.8 \%)$ with positive rapid CPK-MBs. Of these, 19 were admitted to monitored beds. Of the patients discharged, $5(6.5 \%)$ had positive CPK-MBs. Based on follow-up information, a least 3 of these apparently experienced a cardiac event. Of the 82 patients admitted to unmonitored beds, $8(9.8 \%)$ had positive CPK-MBs. Of these, 4 proved to have acute MI's, 2 probably had MI's. and only 2 clearly ruled out for MI.

Conclusion. The rapid CPK-MB assay is reliably reflective of the standard in this setting. Testing ED patients with possible myocardial infarction who are considered. for discharge or unmonitored admission may prevent inappropriate disposition. negative test however, should not be used to formulate disposition decisions because the CPK-MB may not yet have risen. 


\title{
Intravenous magnesium prevents ionized hypocalcaemia during prolonged cardiac arrest and cardiopulmonary resuscitation
}

\author{
C. B. CAIRNS, J. T. NIEMANN \& P. C. D. PELIKAN \\ Harbor-UCLA, Torrance, CA, U.S.A.
}

Free calcium $(\mathrm{Ca}+2)$ has been shown to decrease during prolonged cardiac arrest and resuscitation (CPR). To assess the effect of magnesium $(\mathrm{Mg}+2)$ on free $\mathrm{Ca}+2$ during prolonged arrest and CPR, 14 dogs were subjected to $7.5 \mathrm{~min}$ of $\mathrm{VF}$ followed by $20 \mathrm{~min}$ of conventional CPR. Seven dogs were given $5 \mathrm{mgs} \mathrm{MgSO}_{4}$ immediately prior to begining CPR.

Arterial free $\mathrm{Ca}+2$, blood gases, and lactate were measured at $5 \mathrm{~min}$ intervals. Lactate rose in both groups during the CPR study period (control $1.5 \pm .2 \mathrm{mmol} / \mathrm{L}$ vs $8.4 \pm .6$ and $20 \mathrm{~min}, \mathrm{P}<.05)$. In the untreated group, free $\mathrm{Ca}+2$ decreased during the arrest period (control $4.8 \pm .2 \mathrm{mg} / \mathrm{dl}$ vs $3.4 \pm .1$ at $20 \mathrm{~min}, \mathrm{P}<.05$ ) and a significant correlation between free $\mathrm{Ca}+2$ and lactate was observed $(\mathrm{r}=-.71$, $\mathrm{P}<.001)$. There was no change in total $\mathrm{Ca}+2$. In the $\mathrm{Mg}+2$ group, free $\mathrm{Ca}+2$ did not change significantly during the arrest and CPR period (control $4.9 \pm .2 \mathrm{vs}$ $5.4 \pm .2$ after $20 \mathrm{~min}$ of CPR). CPR arterial pressures and blood gas data were not significantly different between groups.

Conclusions. (1) Free $\mathrm{Ca}+2$ decreases during prolonged $\mathrm{CPR}$ and can be prevented by $\mathrm{Mg}+2$ administration; (2) This effect of $\mathrm{Mg}+2$ is most likely due to $\mathrm{Ca}+2$ channel blockade; (3) Maintenance of a normal free $\mathrm{Ca}+2$ level during arrest and CPR may facilitate restoration of effective spontaneous cardiac function. 


\title{
Oxidative stress is associated with white matter damage resulting from hyperoxic cerebral reperfusion or rapid correction of hyponatraemia
}

\author{
H. S. MICKEL
}

Laboratory of Experimental Neuropathology, NINDS, Laboratory of Biochemistry, NHLBI, National Institutes of Health, Bethesda, Maryland 20892

Myelin is uniquely vulnerable to oxidative stress on account of low levels ơ antioxidant enzymes and highly unsaturated fatty acids present in the myelin sheath. We have demonstrated an association between oxidative stress and myeitite damage in three animal models, two of which are associated with neurologi ity emergencies. These findings indicate that breathing $100 \% \mathrm{O}_{2}$ following cerebra ischemia as well as correcting severe hyponatremia rapidly may be deleterious.

In the Mongolian gerbil, breathing $100 \% \mathrm{O}_{2}$ for $3 \mathrm{~h}$ following $15 \mathrm{~min}$ of globa cerebral ischemia results in selective white matter damage in both the latera corpus striatum and the lateral thalamus as well as a marked increase in lipi $\vec{\phi}$ peroxidation (measured by pentane production) and 14 day cumulative mortality The damage to white matter following hyperoxic reperfusion resembles that foun following the rapid correction of severe hyponatremia in the rat (the rat model fo central pontine myelinolysis or electrolyte-induced myelinolysis, EIM). In EIMP soluble brain protein oxidation occurs by the first day following rapid correction of hyponatremia. Changes in iron and copper metabolism also occur during hyponatremia and/or its correction. It is proposed that these changes are associate with metal-catalyzed protein oxidation, which we measure as free carbonyl groupg associated with the proteins. We will discuss the role of protein oxidation as mechanism of oxidative injury to cells. 


\section{Analysis of emergency medical response during the Loma Prieta Earthquake (San Francisco) - October 7, 1989}

J. J. CALABRO, J. E. POINTER, C. E. SAUNDERS \& J. G. MICHAELIS

University of California, San Francisco School of Medicine, San Francisco EMS Agency

On October 17, 1989, at 17.04 hrs (PDT) an earthquake measuring 7.1 on the Richter scale, lasting $15 \mathrm{~s}$ hit the San Francisco Bay Area, home to more than 5.9 million people. It was felt from Los Angeles on the south to the Oregon-California state line on the north, and easterly to western Nevada. Sections of the San FranciscoOakland Bay Bridge and Interstate 880 collapsed, as did numerous smaller structures in the six county San Francisco Bay Area.

In a 6-month study, a $100 \%$ census questionnaire $(1500)$ was sent to all Basic Emergency Medical Technicians and Paramedics in the six Northern California counties most affected by the Loma Prieta earthquake. Emergency care providers who had significant involvement were identified and interviewed.

Charts of all patients (3000 total) seen at all hospitals (54) in the six counties from $17.04 \mathrm{hrs,}$ October 17 th until $0600 \mathrm{hrs,} \mathrm{October} \mathrm{18th} \mathrm{were} \mathrm{abstracted.} \mathrm{Telephone}$ interviews were completed for all clinics (non-emergency departments) with individual interviews performed with the emergency physicians, emergency nurses and administrators 'in charge' during the earthquake; EMS directors and communications personnel for each EMS Agency. Communications Systems review was divided into reconnaissance, field communications, and base hospital contact.

Preliminary reports show that approximately 3000 patient visits, 63 deaths and over $\$ 6$ billion in property damage could be attributed to the earthquake. Transport of critical patients was done primarily by ambulances with bus, taxi, police, fire, and private vehicles also utilized. Generally, medical personnel were far in excess of that necessary. Field care included very little advanced life support with most transported patients being non-critical. Many of the emergency medical workers were utilized for Search and Rescue operations at the various scenes. Early results show that reconnaissance information was greatly lacking with the local media providing the majority of the information. Field communication was generally adequate although decreased from normal operating patterns. Little or no Base contact was attempted or achieved. Overall, Northern California Emergency Medical 
Systems responded well. However many steps, including training and review of protocols/procedures and equipment have been stimulated by our brush with disaster.

\title{
Cost benefit analysis of EMS services in the treatment of sudden cardiac death
}

\author{
T. VALENZURLA, E. CRISS, D. SPAITE \& \\ H. MEISLIN
}

Section of Emergency Medicine, Arizona Health Sciences Center, University of Arizona, U.S.A.

Hypothesis. The cost benefit relationship per life saved from out-of-hospital sudden cardiac death is comparable to that of other resource intensive medical interventions.

Population. All out-of-hospital, non-traumatic, cardiopulmonary arrests requiring ALS interventions in a medium-sized metropolitan area. Patients were prospectively enrolled over a 10 -month period from $10 / 88$ to $7 / 89$.

Methods. Data were obtained from paramedic encounter forms, dispatch log and real time event recording devices attached to all monitor-defibrillator unitso Age, witnessed $v s$ unwitnessed, time to basic CPR, time to ALS, status on arrivaF to ED and survival status were obtained. An estimate of the cost to maintain the EMS system capable of an $8 \mathrm{~min}$ ALS response time was determined. The cost estimate included: training, personnel, equipment and maintenance. Cost per life saved was compared to that reported for a variety of other medical interventions. Costs were normalized to 1989 dollars.

Results. A total of 190 cardiopulmonary arrests were enrolled. Mean age of victims, 63.9 years. $67 \%$ - male; $33 \%$ - female. A total of $110 / 190(58 \%)-$ witnessed. $46 / 90(24 \%)$ received bystander CPR. Initial rhythm of ventricular fibrillation was found in $79 / 190(42 \%)$. A total of $57 / 190(30 \%)$ found in asystole. A total of $13 / 190(7 \%)$ survived to hospital discharge. EMS system costs per life saved, over the 10-month study period, as compared to other medical interventions:

\begin{tabular}{lc}
\hline \multicolumn{1}{c}{ Intervention } & Cost/life saved -1989 Dollars \\
\hline Bone marrow transplant & $\$ 241000$ \\
Breast cancer screening & 406000 \\
Pertussis vaccine program & 195000 \\
Heart transplant & 109000 \\
Liver transplant & 149000 \\
Sudden cardiac death & 85000 \\
\hline
\end{tabular}


Conclusions. Maintenance of an EMS system in a medium sized metropolitan area capable of successfully resuscitating deaths due to cardiac dysrhythmias compares favorably in cost benefit terms with a varity of medical interventions.

\section{Prehospital pacing of asystole cardiac arrest by paramedics: a controlled clinical trial}

J. R. GRAVES, R. O. CUMMINS, S. HORAN \& M. P. LARSON

Center for Evaluation of Emergency Medical Services, Seattle-King County Department of Public Health and Division of Emergency Medical Services, University of

Washington, U.S.A.

Study hypothesis. We tested whether transcutaneous pacing initiated by paramedics prior to intubation and IV medications can improve survival for patients in primary asystole or post-defibrillation asystole.

Study population. The study population comprised adult patients in non-traumatic cardiac arrest, treated by paramedics.

Methods. The study was a controlled, before-and-after evaluation. Nine paramedics units were trained and authorized to use transcutaneous pacemakers, prior to intubation and IV medications, for patients in primary asystolic or postdefibrillation asystolic cardiac arrest. Data gathered included age, sex, response times, \% witnessed arrest, \% bystander-CPR, admission rates and survival to hospital discharge.

Results. Paramedics treated 554 patients in primary asystole; 330 of these patients were treated according to ACLS protocols, with no pacing; 224 were treated with initial pacing plus standard ACLS protocols. No significant differences were noted between the paced and non-paced groups in age, sex, \% witnessed arrest, \% bystander CPR, and system response times. Time from collapse to initiation of pacing averaged $19 \mathrm{~min}$. In the paced group $13 \%$ (29) were resuscitated and admitted to hospital, compared to $9 \%$ (31) in the non-paced group (NS, P > 0.05). Survival to discharge was $3 \%(7)$ in the paced group and $1 \%(4)$ in the non-paced group (NS). An additional 99 patients in post-defibrillation asystole were paced: $20 \%$ (20) were admitted to hospital, and $4 \%$ (4) survived. Survival rates for these patients were not significantly different from survival rates for either the paced or the non-paced asystolic patients.

Conclusions. Late (19 min after arrest) transcutaneous pacing for primary asystole and post-defibrillation asystole does not significantly improve survival, even when used as the first intervention in ACLS protocols. 


\title{
The distribution of orthostatic vital sign changes in a normal adult population and the variation of these changes with age
}

\author{
D. L. SCHRIGER \& L. J. BARAFF
}

UCLA Emergency Medicine Center and UCLA School of Medicine, California, U.S.A.

Purpose. To determine the distribution of orthostatic vital sign changes (OVSC) in a population of healthy adults thus delineating normal and abnormal $(>+2 \mathrm{SD})$ values.

Hypothesis. The elderly will have a greater increase in pulse and/or a greater decrease in blood pressure upon standing than their younger counterparts.

Study population. Fifty-four healthy adult volunteer blood donors (prior donation) and 100 self-sufficient ambulatory participants at a senior citizen? daytime activity center.

Methods. Each subject was placed supine for at least $1 \mathrm{~min}$ following which their pulse (P) was counted for $30 \mathrm{~s}$ and their blood pressure (BPS/BPD) auscultated in an upper extremity. The patient was then asked to stand for $30 \mathrm{~s}$ and the pulse and BP measurements were repeated in the same arm. Variation in OVSC with age was examined with linear regression. T-tests were used for dichotomous comparisons.

Results. Subjects ranged in age from 19 to 94 (mean 61, SD 22). Mean supine $P$, BPS, and BPD were 72,137 , and 76 . Mean pulse change (PC) was +1 (range -16 to +26 , SD 7). Mean BPS change (BPSC) was +3 (range -30 to +40 , SD 11). Mean BPD change (BPDC) was +3 (range -14 to +28 , SD 8). Mean OVSC in patients $<$ age 65 were: PC +3 , BPSC -1 , BPDC +4 ; and for those $>=65$ were: PC -1 , BPSC +4, BPDC +2 . While mean PC and BPSC differences between adults and elderly were statistically significant $(P<\cdot 001, P<\cdot 005)$, and there was evidence of linear trend with age for each parameter $(P<.02)$; this study was very powerful

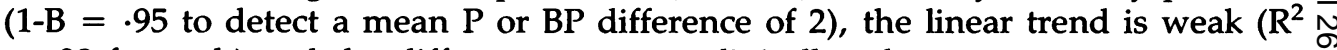
$<.08$ for each) and the differences are not clinically relevant.

Conclusions. Abnormal OVSC, defined as those values $>+2$ S.D. from the mean were: PC $>+15$, BPSC $>-19$, and BPDC $>-13$. There was no clinically meaningful variation of OVSC with age in this study. 


\title{
An evaluation of pulse oximetry in the field
}

\author{
K. AUGHEY, D. HESS, D. EITEL, K. BLEECHER, \\ M. COOLEY \& N. SABULSKY \\ Department of Emergency Medicine, York Hospital, York, U.S.A.
}

Study hypothesis. There is no difference between in-vivo arterial oxygen saturation $(\mathrm{HbO} 2)$ and pulse oximetry saturation $(\mathrm{SpO} 2)$ in the field.

Study population. Adult patients $(n=18)$ in whom there was a clinical indication for arterial blood gases were included in the study.

Methods. A Baxter ASAT pulse oximeter with digital probe was used. Arterial blood was obtained from the radial artery by prehospital providers trained in the technique. The blood was packed in ice water, and analysed promptly by COoximetry after return to the hospital. $\mathrm{SpO} 2$ was recorded at the time the blood was collected. The quality of the oximeter signal was evaluated by observing the plethysmographic waveform. $\mathrm{SpO} 2$ and $\mathrm{HbO} 2$ were compared using correlation analysis, bias (SpO2- $\mathrm{HbO} 2)$ and precision (SD of the differences), and paired student's t-test. In 11 of the patients, carboxyhemoglobin $(\mathrm{COHb})$ and methemoglobin (metHb) were also determined.

Results. There was no significant difference between $\mathrm{SpO} 2(93.3 \pm 6.1 \%)$ and $\mathrm{HbO} 2(93.8 \pm 6.1 \%)(\mathrm{P}=0.38)$. There was a strong correlation between $\mathrm{SpO} 2$ and $\mathrm{HbO} 2(\mathrm{r}=0.93)$. The bias was $-0.5 \%$, with a precision of $2.3 \%$. The mean $\mathrm{COHb}$ was $1.15 \pm 0.85 \%$ and the mean metHb was $0.95 \pm 0.37 \%$.

Conclusions. These preliminary data suggest that pulse oximetry is sufficiently accurate to be useful in the field. Our prehospital providers have developed considerable confidence in the use of pulse oximetry in the field. Further work is needed to evaluate pulse oximetry in settings of elevated $\mathrm{COHb}$ and metHb. 


\title{
Head, facial and clavicular trauma as a predictor of cervical spine injury
}

\author{
J. WILliAMS, D. JEHLE, E. COTTINGTON
}

Allegheny General Hospital, MCP - Allegheny Campus, Pittsburgh, U.S.A.

Study hypothesis. The American College of Surgeons teaches that 'trauma occurring above the clavicle should raise a high suspicion for a potential C-spine injury'. In this study, we investigated the association of head, facial and clavicular trauma with cervical spine and cord injury.

Methods. The records of 5021 consecutive trauma patients admitted to a level I Regional Trauma Center over a $3 \frac{1}{2}$-year-period were retrospectively reviewed. The incidence of head, facial, clavicle, cervical spine and cervical cord injuries were recorded. Glascow Coma Scores (GCS) were obtained on all patients. Statistica analysis using multiple logistic regression and Chi-Square analysis was performed to determine the relationship between traumatic injury above the clavicle and cervical spine and cord injury.

Results. Head injured patients had no greater incidence of cervical spine injury than non-head injured patients $(4.76 \%$ vs $4.37 \% \mathrm{P}=0.52)$ but were found to have significantly fewer spinal cord injuries $(1.5 \%$ vs $2.3 \% \mathrm{P}=0.048)$. There was no difference in incidence of cervical spine injuries between patients with and without facial injuries, $(4.2 \%$ vs $4.6 \% \mathrm{P}=0.61)$. However, there were significantly fewer cord injuries among patients with facial injuries $(0.75 \%$ vs $2.2 \% \mathrm{P}=0.01)$. The presence or absence of clavicular fracture was not associated with a significant increase in cervical spine $(6.9 \%$ vs $4.4 \% \mathrm{P}=0.11)$ or cervical cord injuries $(1.6 \%$ vs $2.0 \% \mathrm{P}=0.68)$. A GCS of $<14$ was associated with a higher incidence of $\mathrm{C}$-spine injury than GCS $\geq 14$ in both head injured $(6.7 \%$ vs $3.9 \% \mathrm{P}=0.007)$ and non-head injured patients $(12.2 \%$ vs $5.9 \% \mathrm{P}=0.002)$. There was a greater incidence of cervical cord injury among patients with GCS $<14$ vs GCS $\geq 14$ in both the head injured $(2.2 \%$ vs $1.2 \% \mathrm{P}=0.09)$ and non-head injured $(8.8 \%$ vs $2.7 \% \mathrm{P}<0.0001)$ groups.

Conclusions. Trauma to the head, face and clavicle is not associated with a higher incidence of cervical spine or cord injury. Physiologic parameters such as the GCS appear to be more accurate predictors of cervical spine or cord injury than mere evidence of 'trauma occurring above the clavicle'. 


\title{
Effects of $15 \%$ sodium chloride during uncontrolled continuous haemorrhage
}

\author{
P. F. VAN LigTEN, C. J. LESLIE, J. W. HOEKSTRA, \\ R. F. GRIFFITH \& C. G. BROWN \\ Division of Emergency Medicine, The Ohio State University, Columbus, U.S.A.
}

Study hypothesis. Shock resuscitation during uncontrolled continuous haemorrhage (UCH) with a small volume infusion of $15 \%$ hypertonic saline solution (HTS) is superior to isotonic Lactated Ringer's solution (LR).

Study population. Eight swine weighing $>20 \mathrm{kgs}$, LR $(n=4)$, HTS $(n=4)$.

Methods. UCH was initiated in splenectomized swine. At $25 \mathrm{~min}$ of haemorrhage, the animals were ventilated and randomly treated with $4 \mathrm{ml} / \mathrm{kg}$ (rate of $1 \mathrm{ml} / \mathrm{kg}$ / min) of HTS or LR. After receiving the study solution, both groups received a constant infusion of LR. Physiologic data sets were recorded at $10 \mathrm{~min}$ intervals. Data were analysed using analyses of variance and student's t-tests.

\begin{tabular}{lrrrrrrr}
\hline Results [means]: & LR & \multicolumn{7}{c}{ HTS } & & P \\
\hline Time (min) & 20.0 & 30.0 & 40.0 & 20.0 & 30.0 & 40.0 & \\
CI (1/min/M ${ }^{2}$ ) & 1.9 & 1.6 & 0.8 & 2.3 & 3.4 & 2.5 & 0.01 \\
LV stroke Work (gmM/M ${ }^{2}$ ) & $7 \cdot 8$ & 4.7 & 2.0 & 11.0 & 14.2 & 9.2 & 0.01 \\
Oxygen Extraction (\%) & 77.0 & 80.0 & 82.0 & 71.0 & 39.0 & 70.0 & 0.006 \\
MAP (mmHg) & 50.0 & 46.0 & 31.0 & 50.0 & 49.0 & 47.0 & 0.49 \\
Total Bleed Volumes (ml) & & & 988.0 & & & 1160.0 & 0.39 \\
\hline
\end{tabular}

Conclusion. The data suggest that $15 \%$ HTS significantly improves cardiac performance in shock due to uncontrolled continuous haemorrhage, compared to isotonic LR alone. No exacerbation of haemorrhage rate was evidenced, presumably because mean arterial pressure was minimally altered. 


\title{
AMI detection in chest pain patients with non-diagnostic ECG's: serial CK-MB sampling in the ED
}

\author{
W. B. GIBLER, G. P. YOUNG, J. R. HEDGES, \\ L. M. LEWIS, M. S. SMITH, R. AGHABABIAN, \\ R. O. JORDEN, E. J. ALLISON, E. J. OTTEN \& \\ P. K. MAKENS
}

University of Cincinnati Medical Center, Ohio, U.S.A.

Study hypothesis. Serial CK-MB sampling in the ED can identify acute myocardial $\stackrel{\mathbb{D}}{\stackrel{2}{*}}$ infarction (AMI) in patients presenting to the emergency department (ED) with chest pain and non-diagnostic electrocardiograms (NDECG).

Study population. Patients ( $>30$ years hospitalized for chest pain at six differe medical center hospitals.

Methods. This prospective study assessed serial CK-MB levels using a rapid serum assay (Tandem ${ }^{\mathrm{TM}}$ ICON QSR CK-MB Assay, Hybritech Inc.) for identification of AMI patients with NDECGs. Levels were determined upon presentation and hourly for $3 \mathrm{~h}$ following ED presentation. Patients with rising levels for at least three time intervals or one level $>7 \mathrm{ng} / \mathrm{ml}$ were considered to have a positive enzyme study. ECGs showing $\geq 1 \mathrm{~mm}$ ST-elevation in two contiguous leads were considered to be 'diagnostic'. AMI was determined by the development of new Q-waves or standard in-hospital enzyme changes.

Results. Of the 692 patients, 104 were considered to have an AMI; 60 of these had NDECGs. Of patients with NDECGs, 52 (87\% sensitivity) had a positive enzyme study within $3 \mathrm{~h}$ following presentation. The predictive value of a negative enzyme study for no AMI was $\mathbf{9 6 \%}$ (specificity $95 \%$ ). Combining serial ED CK-MB 9 assay results with diagnostic ECGs yielded a $92 \%$ sensitivity for AMI detection.

Conclusions. Serial CK-MB determination in the ED can help identify patients with AMI and NDECGs. Since nearly $50 \%$ of patients with AMI present to the ED with NDECGs, use of serial CK-MB analysis should facilitate optimal in-hospital $N$ disposition and promises to help guide therapeutic interventions in patients with suspected AMI. 


\title{
Racemic epinephrine in the treatment of laryngotracheitis: Can we identify patients for outpatient management?
}

\author{
D. HARTMAN, M. PRENDERGAST \& J. JONES \\ Emergency Medicine Residency Program, Butterworth Hospital, Michigan State \\ University College of Human Medicine, Grand Rapids, Michigan, U.S.A.
}

Study hypothesis. Objective clinical scoring may be used to identify children with laryngotracheitis (croup) who may be safely discharged from the ED following treatment with nebulized racemic epinephrine and prolonged observation.

Study population. All patients presenting to the ED between March and December 1989 with the diagnosis of laryngotracheitis were evaluated clinically according to a croup scoring system. Twenty-eight patients (aged 6-78 months) with persistent inspiratory stridor at rest after $20-30 \mathrm{~min}$ of mist therapy received nebulized racemic epinephrine $(\cdot 05 \mathrm{cc} / \mathrm{kg}$ of a $2 \cdot 25 \%$ solution) and were enrolled.

Methods. Patients were observed in the ED for a period of $3 \mathrm{~h}$ while clinical scores were assessed at 15, 60, 120, and $180 \mathrm{~min}$ following treatment with racemic epinephrine. Subsequent admission or discharge from the ED was at the discretion of attending physicians and did not follow any preplanned protocol. Clinical variables were analysed (Student's t-test) to identify significant differences between the two groups. Discharged patients were followed up by telephone.

Results. Clinical scores were significantly improved $(\mathrm{P}<\cdot 01)$ throughout the observation period in 21 patients $(75 \%)$ who were discharged from the ED. Only one patient returned within $24 \mathrm{~h}$ for further cool mist therapy. The 7 patients requiring admission to the hospital were younger (14.4 vs 26.2 months) and had higher pretreatment croup scores $(6 \cdot 1$ vs $4 \cdot 1)$.

Conclusions. These results identify a subset of children with croup who may be safely discharged from the ED following treatment with racemic epinephrine. 


\title{
Real time ultrasound for the detection of deep venous thrombois
}

\author{
R. D. POWERS, J. F. CHANCE, P. ABBITT \& \\ C. TEGTMEYER
}

Division of Emergency Medicine and Department of Radiology, University of Virginia Health Sciences Center, Charlottesville, U.S.A.

Study hypothesis. Real-time ultrasound (US) can accurately detect deep venous thrombosis of the leg in emergency department patients.

Population. Seventy adults presenting to a University hospital ED with clinical signs and symptoms consistent with DVT.

Methods. All patients had US of the involved leg followed by contrast venography $\vec{\emptyset}$ Results were sequentially made available to ED clinicians, who determined the course of therapy after both test results were known.

Results. Ultrasound detected DVT in 14 patients $(20 \%)$, all involving thigh veins. All were confirmed by venography. Venography detected an additional six patients with calf DVT who had normal ultrasound studies. All 20 patients with DVT were hospitalized for anticoagulation. Reliance on US alone would have missed 6/20 $(33 \%)$ cases of DVT, but no cases of DVT involving veins proximal to the knee.

Conclusion. As an ED screening test for DVT, US is sensitive for thrombosis in thigh veins, but cannot visualize venous structures distal to the knee. Clinicians can utilize US to reliably diagnose or exclude thigh DVT but venography is necessary to determine whether thrombosis is present in calf veins. 


\section{Outcome of patients discharged from the emergency department with nonspecific abdominal pain}

G. R. COX, J. SCOTT, S. SANFORD \& D. ROSELLE

Department of Emergency Medicine, George Washington University Medical Center, and the Georgtown/George Washington University Emergency Medicine Residency Program, Washington, U.S.A.

Study objective. To determine the subsequent course and outcome of patients discharged from the ED with abdominal pain of uncertain etiology.

Study population. Cohort study of 385 consecutive patients over the age of 16 seen in an inner-city university hospital ED and discharged with the diagnosis of 'nonspecific abdominal pain' or 'abdominal pain, uncertain etiology'. A total of 118 were lost to follow-up, resulting in a final study population of 267 patients.

Methods. Telephone contact 2 days and 2 months after the ED visit to obtain information regarding symptoms, subsequent evaluation, and final diagnosis.

Results. Two month follow-up was completed on 209 females (78.3\%) and 58 males $(21.7 \%)$ with a median age of 30 (range $16-78$ ). The majority $(58.4 \%)$ presented with lower abdominal pain. Symptoms at 2-day follow-up had resolved in 115 patients $(43.1 \%)$ and did not recur in 90 of these (33.7\%). At 2 months pain had resolved in 177 patients $(66.3 \%)$, continued intermittently in $78(29.2 \%)$ and remained constant in $12(4.5 \%)$. Nine patients had undergone surgery. No diagnosis was established in 225 patients (84.3\%). Twenty-one of 42 patients in whom a cause for abdominal pain was identified were women with pelvic pathology.

Conclusions. Most patients with abdominal pain in whom a diagnosis can not be established in the ED experience resolution of symptoms without sequelae. In the vast majority, a firm diagnosis will not be determined despite further evaluation. The most common missed problems are pelvic disorders in women. 


\title{
A prospective evaluation of liver function tests in screening for intra-abdominal injury
}

\author{
R. SAHDEV, R. J. SCHWARTZ, R. R. GARRAMONE
} \& L. M. JACOBS

Department of Emergency Medicine/Trauma, Hartford Hospital, Hartford, Ct. and University of Connecticut, School of Medicine, Farmington, U.S.A.

Study objective. To determine the screening value of LFTs (SGOT, SGPT) for intraabdominal (IA) injury in blunt trauma.

Methods. Three-hundred and eight consecutive adult blunt trauma patients (pts) with admission LFTs were prospectively studied during 9 months at a level I facility. IA injury was diagnosed by CT or laparotomy (lap). Patients were fol- $\vec{\bullet}$ lowed until discharge.

Results. Age average was 35 years, TS 14 and ISS 13 . Mortality was $5.3 \%$. patient had a missed abdominal (abd) injury. Statistical analysis showed LFTs of $130 \mathrm{IU}$ to have the highest sensitivity, and lowest false positive rate.

\begin{tabular}{lcccc}
\hline $\begin{array}{l}\text { LFT's } \\
\text { IU/1 }\end{array}$ & $\begin{array}{c}\text { Total } \\
\text { Pts }\end{array}$ & $\begin{array}{c}\text { Pts with } \\
\text { CT or Lap }\end{array}$ & $\begin{array}{c}\text { Total } \\
\text { Liver Injury }\end{array}$ & Total IA Injury \\
\hline$<30$ & 85 & $22(26 \%)$ & $0(0 \%)$ & $5(6 \%)$ \\
$<130$ & 250 & $96(38 \%)$ & $0(0 \%)$ & $21(8 \%)$ \\
$<130$ & 58 & $45(78 \%)$ & $18(100 \%)$ & $30(52 \%)$ \\
\hline
\end{tabular}

Higher LFT values had an increased incidence of liver injury. Ninety per cent of patients with LFTs $>500(n=10)$ had liver injury. Other IA injuries increased3 significantly at LFTs of $>130$ (Table, $P=<\cdot 001$ ). Four patients with liver injuryo not requiring repair underwent a lap based on a positive DPL. All 4 had LFTS $>130$. Of 11 patients with IA injury and a normal abdominal exam, 10 hade. elevated LFT's.

Conclusions. 1) LFTs are a marker for significant IA injury; 2) Stable patientsin with LFTs $>130$ should have and evaluation by CT to decease false positive DPLs; 3) LFTs on admission of $<130 \mathrm{IU} / 1$ exclude liver injury. 


\section{Faculty attrition among three specialties}

H. THOMAS, J. C. MOORHEAD \&

A. R. MAGNUSSON

Bowman Gray School of Medicine, North Carolina and The Orgeon Health Sciences University, Oregon, U.S.A.

Study hypothesis. Many Emergency Medicine (EM) physicians perceive that more of their colleagues leave academic medicine than physicians in other medical disciplines.

Study population. All institutions with residencies in EM were surveyed.

Methods. Questions were asked pertaining to the year 1988, including size of faculty, number of physicians leaving, reasons for leaving, total hours worked, and night-time hours worked. Answers were compared with those from Orthopedics (ORT) and Cardiology (CAR) depts. From the same institutions.

Results. Reports were returned from 67 of 68 (98.5\%) EM, 53 of 58 (91.4\%) ORT, and 47 of $54(87 \%)$ CAR depts. Among 670 total EM physicians (average faculty size 10$), 67(10 \%)$ had left: $38(56.7 \%)$ to enter private practice, $18(26.8 \%)$ to another academic position, and $11(16.4 \%)$ to retire, change specialty, etc. There were no significant differences (Mantel-Haenzel test, $P=.78$ ) between those findings and data from the ORT [total physicians - 379; average number $-7 \cdot 1$; $30(7.92 \%)$ having left] and the CAR departments [total physicians - 659; average number - 14; $58(8.8 \%)$ having left]. EM physicians average $46 \mathrm{~h} /$ week, significantly fewer $(P=.001)$ than those of ORT $(60.6 \mathrm{~h})$ or CAR $(56.9 \mathrm{~h})$. EM physicians did work significantly more night-time hours (6.96/week) versus $4 \cdot 33$ /week ORT and 3.33/week CAR $(P=\cdot 03)$.

Conclusion. This comparison of three similar medical disciplines failed to show a higher attrition rate for EM faculty. 


\title{
Is maxillofacial injury an indicator of cervical
} spine injury in children?

\author{
K. F. O'MALlEY, S. E. ROSS \& R. SWEENEY
}

Division of Trauma and Emergency Medical Services, UMDNJ/Robert Wood Johnson Medical School at Camden, Cooper Hospital/University Medical Center, U.S.A.

The incidence of cervical spine injury has been reported to be increased in the 9 presence of significant maxillofacial injury in adults. To investigate the validity of $\overrightarrow{-}$ this hypothesis in the paediatric population, a retrospective review of 580 patients below the age of 17 admitted to a Level I trauma centre between 1/1/86 and $6 / 30 / 89$ was performed.

The mean age was $8 \cdot 1$, mean Injury Severity Score (ISS) was 13.7 , and there were 29 deaths $(5 \%)$. There were 4 patients $(0.7 \%)$ with injury to the cervical spine, âd considered to be unstable (AIS $>3$ ). Thirty-six patients $(6 \cdot 2 \%)$ had suffered maxillofacial injuries carrying an Abbreviated Injury Scale (AIS) rating of 2 §r greater (17 AIS-2 and 19 AIS > 2), none of whom was found to have injury to the cervical spine.

We conclude that significant maxillofacial injury does not appear to be associated $\stackrel{\mathscr{Q}}{\circ}$ with an increased incidence of injury to the cervical spine in the pediatric age $\overrightarrow{\vec{\sigma}}$ group. Although the possibility of such injury must be considered, there should be no hesitation in performing definitive airway control maneuvers due to fear of precipitating or aggravating damage to the cervical spinal cord on this basis. 


\title{
Key role of prehospital resuscitation in survival from out-of-hospital cardiac arrest
}

\author{
M. J. BONNIN, P. E. PEPE \& P. S. CLARK \\ Department of Medicine, Boylor College of Medicine and City of Houston Emergency \\ Medical Services, Houston, Texas, U.S.A.
}

In view of the inherent hazards of emergency transport, we examined the value of continued ED resuscitative efforts for non-traumatic out-of-hospital cardiac arrest patients (OHCAPS) when prehospital advanced life support (ALS) fails to achieve restoration of spontaneous circulation (ROSC).

For 16 months, all OHCAPS were prospectively studied in terms of multiple factors including the timing of ALS procedures, ROSC, in-patient admission (ADM), and successful hospital discharge.

Results: Of the 1283 OHCAPS studied, 909 did not attain prehospital ROSC. Despite further efforts at the ED, only 18 of these $(2 \%)$ achieved ADM and only two $(0 \cdot 2 \%)$ were eventually discharged. Both survivors had presented and remained in ventricular fibrillation (VF) throughout the prehospital phase and both were left with residual neurological deficits. During the study period, the overall discharge rate for VF cases was about $20 \%$ while mean ALS scene/transport times were 27.8 and $7.8 \mathrm{~min}$.

Conclusion. With the sole possible exception of refractory VF cases, these data support the validity of terminating resuscitative efforts at the scene when patients do not regain pulses following standard ALS interventions. 


\title{
Soft tissue infections in the emergency department: the case for 'simple' antibiotic
}

\section{use}

\author{
R. D. POWERS
}

Division of Emergency Medicine, Department of Medicine, University of Virginia Health Sciences Center, Charlottsville, VA, U.S.A.

Study hypothesis. Soft tissue infections (STI) in ED patients are caused by predictabled pathogens, and do not require newer, broader spectrum, and more expensive antibiotics for effective treatment.

Population. Seventy-two adult University Hospital ED patients with STI (abscess, cellulitis, wound infection, impetigo) appropriate for outpatient treatment widige oral antibiotics.

Methods. Healthy volunteers with STI were enrolled in a prospective, randomizedd trial comparing 10 days of therapy with cephalexin $(500 \mathrm{mg}$ BID or OID) or ofloxacin ( $300 \mathrm{mg}$ or $400 \mathrm{mg} \mathrm{BID}$ ). Ofloxacin (Ortho Pharmaceuticals) is an oral quinolone with a spectrum similar to ciprofloxacin. Microbiology and clinical parameterso were assessed in initial and two follow-up visits.

Results. Forty-eight $(67 \%)$ of patients had cultures positive for pathogens. Of these, $80 \%$ were staph or strep species with routine sensitivities. Gram negative rods were isolated in only 5 patients. There was no difference in outcome between the cephalexin and ofloxacin groups, with clinical response rates exceeding $95 \% \stackrel{3}{3}$. Patients with no identifiable pathogen responded as well as those with positive cultures.

Conclusions. STI in ED patients are not caused by exotic or multiply resistant flora. The bacteriologic profile and clinical course of ED STI indicate that treatmento with a 'simple' antibiotic, such as cephalexin, will be effective in nearly all cases.? Empiric use of broader spectrum, often costlier antibiotics for initial therapy of uncomplicated ED STI cannot be justified. 


\section{Performance and interpretation of pelvic ultrasound by emergency physicians in patients with suspected ectopic pregnancy: a prospective study}

L. M. GUSSOW \& G. ALBERTO

Department of Emergency Medicine, Cook County Hospital, Chicago, Illinois, U.S.A.

Study hypothesis. Emergency physicians can use ultrasound imaging to reliably establish the presence of intrauterine pregnancy (IUP) in patients with suspected ectopic pregnancy.

Study population. Emergency Department patients with suspected ectopic pregnancy. All had been scheduled for formal emergency pelvic ultrasound in the Radiology or Obstetrics department.

Methods. Two Emergency physicians received a maximum of $6 \mathrm{~h}$ training in pelvic ultrasound, supplemented by individual reading. Patients entered into the study had their bladders filled by the technique indicated for the formal test. In a blinded manner, one of the two authors performed a trans-abdominal pelvic scan on each patient, using the ScanMate II portable ultrasound scanner (Damon Corp.) fitted with a $3.5 \mathrm{MHz}$ probe. The scan was interpreted immediately, with definite IUP being established by the visualization of a yolk sac, fetal pole, fetal heart movement, or double decidual sign. This reading was compared to the formal scan by Radiology or Obstetrics.

Results. Seventeen patients were studied. The average patient age was 23.4 years; the average gestational age was 8.1 menstrual weeks. Seven scans were read by the Emergency physician as demonstrating IUP; all of these were confirmed by the formal scan $(P<\cdot 01)$. Ten scans were read by the Emergency physician as not demonstrating a definite IUP; all of these readings agreed with the formal scan $(\mathrm{P}<\cdot 001)$.

Conclusions. Emergency physicians can reliably perform pelvic ultrasound in patients with suspected ectopic pregnancy, and interpret the results as to the presence or absence of definite intrauterine pregnancy. 


\section{Selective aortic arch perfusion: description o a technique with potential benefit in cardiopulmonary and cerebral resuscitation}

J. E. MANNING, C. A. MURPHY Jr, C. M. HERTZ,

S. G. PERRETTA, R. A. MEULlER \& E. A. NORFLEET

Department of Surgery, Division of Emergency Medical Services and Department of Anaesthesiology, University of North Carolina at Chapel Hill, School of Medicine, North Carolina, U.S.A.

Hypothesis. Brief selective aortic arch perfusion (SAAP) with a cardio-cerebra $\stackrel{\mathbb{1}}{\mathrm{L}}$ protective solution may promote successful resuscitation.

Study objective. To demonstrate that SAAP is technically feasible.

Methods. Five anesthetized dogs $(25-35 \mathrm{~kg})$ had mid-aortic arch pressure (AoAr市) right atrial pressure (RAP), and large-lumen distal aortic arch balloon catheters placed by fluoroscopy. A left carotid artery electromagnetic flow (CEMF) probeڤ was placed. After $10 \mathrm{~min}$ of ventricular fibrillation, the balloon was inflated to $250 \mathrm{mmHg}$ and $29 \pm 4 \mathrm{ml} / \mathrm{kg} / \mathrm{min}$ of $0.9 \% \mathrm{NaCl}$ was infused into the aortic arch for $\overrightarrow{0}$ $2 \mathrm{~min}$ by a Sarns pump. AoArP (mmHg), RAP (mmHg), and CEMF were recorded before (pre-I), during (I) and after infusion. Data (Mean $\pm \mathrm{SD}$ ) were analysed by paired t-test.

Results. AoArP and RAP rose demonstrating arterio-venous flow (Table).

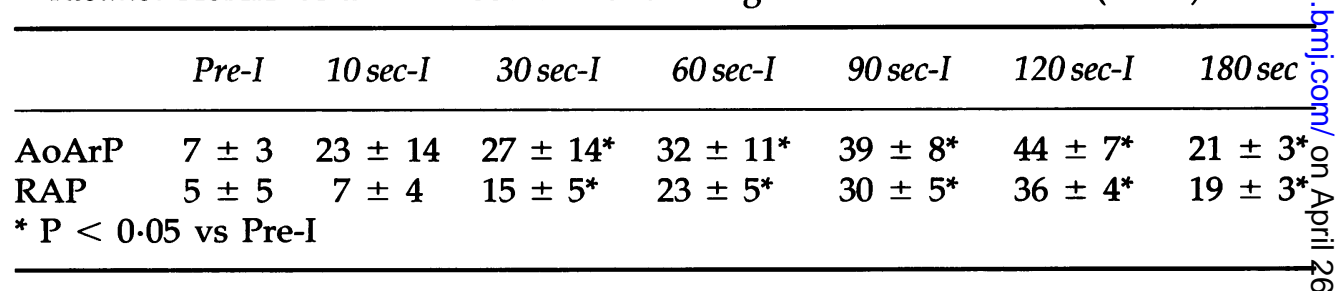

CEMF averaged $50 \pm 16 \%$ of pre-arrest $(\mathrm{P}<0.05$ vs Pre-I). Small AoArP-RAP differences despite good flow rates suggested low vascular resistance. Contrast్ showed aortic valve closure and minimal leak past the balloon.

Conclusion. SAAP is technically feasible. Solutions improving cardiac and cerebrab function could be delivered by SAAP during cardiac arrest. 


\section{Comparison of coronary perfusion pressure using radial and central aortic pressures during CPR in humans}

E. P. RIVERS, J. LOZON, N. A. PARADIS \& M. G. GOETTING

Department of Emergency Medicine, Henry Ford Hospital, Detroit, Michigan, U.S.A.

The Central aortic (Ao) to right atrial pressure $(\mathrm{Ra})$ gradients during CPR relaxation phase is the coronary perfusion pressure (CPR) and correlates with the return of spontaneous circulation.

Hypothesis. Radial artery and femoral artery pressure may be a convenient substitute for Ao pressure in calculating the CPP.

Population. Forty-five normothermic, cardiac arrest patients.

Methods. While receiving advanced cardiac life support, right atrial (45 pts.), Ao (45), Radial (45), and Femoral (17) catheters were inserted. Pressures were measured simultaneously at baseline, and after administration of 1 and $14 \mathrm{mg}$ of epinephrine. Pressures reported represent maximum Aortic (Ao), Radial (Rad), and Femoral (Fem) pressure response to epinephrine.

Results. CPP gradients

\begin{tabular}{lcrr}
\hline & $\begin{array}{c}\mathrm{T}=0 \text { (baseline) } \\
\text { Mean }(S D)\end{array}$ & $\begin{array}{c}\mathrm{T}=1(1 \mathrm{mg}) \\
\text { Mean }(S D)\end{array}$ & $\begin{array}{c}\mathrm{T}=2(14 \mathrm{mg}) \\
\text { Mean }(S D)\end{array}$ \\
\hline Ao-Ra & $4.97(9.4)$ & $9.10(9.5)$ & $13.4(9.8)$ \\
Rad-Ra & $6.30(8.5)$ & $10.1(9.5)$ & $14.9(10.5)$ \\
Fem-Ra & $4.32(4.3)$ & $9.10(6.7)$ & $14.3(8.5)$
\end{tabular}

All radial pressures except $T=1$ relaxation phase were statistically different from aortic. There were no statistical differences between aortic and femoral pressures. However, correlation between aortic and radial relaxation pressures were 0.96 , 0.94 , and 0.98 at $\mathrm{T}=0,1$, and 2 respectively. Femoral relaxation phase correlations were $0.97,0.96,0.98$ for $\mathrm{T}=0,1$, and 2 respectively. Four patients are not reflected in this data as a result of uninterpretable radial waveforms.

Conclusion. Radial relaxation phase pressure, although slightly higher, correlates with aortic relaxation phase pressure response to vasopressor therapy during CPR. 
Femoral relaxation phase pressure also correlates with aortic relaxation phase pressure during CPR. CPR obtained with radial and femoral arteries is useful in measuring response to vasopressor therapy during CPR when an interpretable? waveform exists.

\title{
Diagnostic peritoneal lavage in abdominal
} stab wounds: analysis of the red blood count via receiver-operating-characteristic curve

\author{
M. J. ZAPPA, A. L. HARWOOD-NUSS, \\ R. L. WEARS \& W. F. FALLON
}

Division of Emergency Medicine, Department of Surgery, University of Florida, Jacksonville, U.S.A.

Study hypothesis. The optimal cut off of red blood cells (RBC) for diagnosicas peritoneal lavage (DPL) in penetrating abdominal trauma can be objective y. determined.

Study population. Ninety-one consecutive adults with abdominal stab wounds not meeting criteria for immediate laparotomy underwent DPL.

Methods. Patients were identified by retrospective review of the trauma log ando trauma registry, and placed in two groups. Group I underwent laparotomy and $\overrightarrow{\overrightarrow{\vec{P}}}$ had findings which required surgical intervention; Group II consisted of those who underwent laparotomy but did not require surgical intervention and those who had no surgery, with a benign hospital course and follow-up. ReceiverOperating-Characteristic (ROC) analysis was done on the DPL RBC count for these two groups.

Results. Overlap between the groups was minimal with the 25th percentile of the $\mathrm{RBC}$ count in Group I being $109000 / \mathrm{mm}^{3}$ and the 75th percentile of the RBC counto in Group II being $478 / \mathrm{mm}^{3}$. The area under the ROC curve generated for these data was 0.95 , implying that the RBC count is a good discriminator. If the prob-9 ability of having an injury which required surgical intervention were equal to that of having an injury not requiring surgical intervention, the cut off RBC count for minimum misclassification (calculated by computing the slope of the fitted curve) would be $5000-10000 / \mathrm{mm}^{3}$. With the observed probability of $25.3 \%$ of abdominals stab wounds requiring surgical intervention, the cut off RBC count for maximume accuracy is $20000 / \mathrm{mm}^{3}$.

Conclusion. An RBC count of $20000 / \mathrm{mm}^{3}$ is likely to be most discriminating between those patients requiring surgical intervention and those not (kappa $\cdot 861, \stackrel{\infty}{\rightarrow}$ $\mathrm{P}<\cdot 001)$. 


\title{
Reduction of medications on paramedic ambulances
}

\author{
J. C. JOHNSON \& G. L. ATHERTON
}

Porter Memorial Hospital, Indiana University School of Medicine, University of Chicago Hospitals, Illinois, U.S.A.

Study hypothesis. The number and types of medications available for prehospital usage by paramedics should be reduced.

Study population. Two thousand four hundred fifty-nine patients requiring paramedic intervention at the ALS level were included in the study population. Non-emergency and non-ALS patients were excluded.

Methods. Retrospective review of patient charts (ambulance \& ED) for calendar year 1989.

Results. Sixty-seven percent of the patients were monitored, required IV therapy and did not require medication. Thirty-three percent of the patients required one or more medications or dosages of medication in addition to IV therapy. Fifty-four percent of the dosages administered involved 5 of the 24 medications available. Two medications were never used and 12 other medications were used less than 20 times during the year.

Conclusions. The difficulty in retaining knowledge related to medications and procedures infrequently utilized is a recurrent theme in medicine. Elimination of medications (infrequently utilized or not utilized), from the current paramedic armamentarium will streamline prehospital protocols, reduce educational needs (primary \& continuing), potentially reduce medication errors and certainly reduce costs relative to stocking and replacement on prehospital vehicles. 


\title{
Hospital and emergency department overcrowding: results of a national survey
}

\author{
A. KELLERMANN, D. ANDRULIS \& B. HACKMAN \\ University of Tennessee, Memphis and National Public Health $\mathcal{E}$ Hospital Institution, \\ U.S.A.
}

Emergency department overcrowding has received increasing media attention in the past 18 months, but the extent and distribution of this problem nationwide is unknown. To assess the situation in our nation's major emergency and trauma care institutions, we surveyed, in the fall of 1988, member institutions of the National Association of Public Hospitals and the Council of Teaching Hospitals ( $\mathrm{N}=465$ total). Two mailings and phone follow-up yielded 277 replies ( $60 \%$ response rate). More than half of responding institutions $(56 \%)$ are privately owned; $44 \%$ are public or veterans hospitals.

Medical school affiliation was noted by $86 \%$. Eighty-two support emergency medicine residency programs. Overall, $40 \%$ of in-patients at these hospitals are admitted through the ED, though at $\frac{1}{4}$ of public hospitals ED patients make up $70 \%$ or more of total admissions. Three-fourths of responding hospitals report increased ED utilization over the past 3 years. Mean occupancy rates would not suggest crowding is a problem: only 17 private and 9 public hospitals reported mean rates of $90 \%$ or more. However, 'holding' of admitted ED patients due to periodic crowding is common: over $\frac{1}{4}$ of responding hospital ED's hold admitted patients a mean of $\geq 4 \mathrm{~h}$ after they are ready for transport to a room. Periodic waits $\geq 12 \mathrm{~h}$ were nc'ed by $36 \%$ of hospitals; $25 \%$ occasionally hold admitted floor patients $24 \mathrm{~h}$ or more. Access to critical care is also difficult: $\frac{1}{5}$ of responding hospitals report mean ICU patient waits of $\geq 4 \mathrm{~h} ; 27 \%$ periodically hold ICU patients $\geq 12 \mathrm{~h}$ and $19 \%$ periodically hold critically ill or injured patients $\geq 24 \mathrm{~h}$. In response to overcrowding, $30 \%$ of responding hospitals refuse interhospital transfers, 33\% transfer patients to other hospitals, $40 \%$ divert selected ambulance patients and $22 \%$ periodically divert all ambulances. Despite such efforts, the cost of ED crowding is high. At $65 \%$ of these hospitals, ED directors feel that ED overcrowding is having a moderate to severe adverse impact on the quality of their emergency care. 


\title{
Under-reporting of contaminated needlestick injuries in emergency health care workers
}

\author{
D. TANDBERG, K. K. STEWART \& D. DOEZEMA
}

University of New Mexico, School of Medicine, Division of Emergency Medicine, Albuquerque, New Mexico, U.S.A.

Study hypothesis. There is considerable under-reporting of contaminated occupational needlestick among emergency health care workers.

Population. A convenience sample of 259 emergency physicians, nurses, and EMTs.

Methods. A survey instrument eliciting demographic and work-related factors was developed and administered. Survey items included age, sex, occupation, years in occupation, the number of procedures performed per week, the number of contaminated needlestick (and other 'sharps') injuries experienced, and the number formally reported during the previous 5 years. Nonsegmented visual analogue scales were used to assess eight attitudes possibly associated with nonreporting. Analysis was by ANOVA and multiple linear regression with stepwise variable selection.

Results. Subject experienced 643 contaminated exposures during the 5 year study reriod, but only 228 (35\%) were formally reported. One or more injuries occurred in $55 \%$ of EMTs, compared with $72 \%$ of nurses and $80 \%$ of MDs $(P<.05)$. Physicians experienced a mean of 3.83 contaminated exposures, while nurses recalled 2.82 and EMTs only $1.75(P<.05)$. MDs formally reported a mean of 0.26 exposures, while EMTs reported 0.85 and nurses reported $1.25(\mathrm{P}<.05)$. MDs formally reported only one eighth of their injuries compared to EMTs and nurses, who reported two thirds of these events $(P<\cdot 05)$. Perception of risk, occupation, years in occupation, and concern about paperwork were the most powerful predictors of reporting rate $(\mathrm{P}<\cdot 05)$.

Conclusions. Work-related contaminated needlestick injuries are under-reported by emergency health care workers, especially emergency physicians. 


\section{Is the anteroposterior radiograph of the cervical spine}

C. J. HOLliMAN, J. S. MAYER, R. T. COOK Jnr. \& J. S. SMITH Jnr.

University Hospital, The Milton S. Hershey Medical Center, The Pennsylvania State University, Pennsylvania, U.S.A.

Study hypothesis. The anteroposterior radiograph of the cervical spine is not needed as a routine screening film for the evaluation of the patient with potential cervical spine trauma if the standard crosstable lateral and open-mouth odontoid views are satisfactorily performed.

Study population. The trauma case registry was utilized and the charts of patients with discharge or postmortem diagnoses of cervical spine fracture cervical spinal cord injury treated at a Level 1 trauma center over a 3-year period were reviewed. A total of 48 patients were studied.

Methods. A faculty neuroradiologist (without knowledge of the original film interpretations) examined the cervical spine films for each case. Sequentially the crosstable lateral film, then the open-mouth odontoid film, and then the anteroposterior film were examined to determine if additional information about the presence of spine injury was gained from each film. Additional studies obtained (obliques, tomograms, etc.) were also reviewed.

Results. In no case did the anteroposterior view provide useful information or demonstrate an injury not apparent on the lateral or open-mouth views.

Conclusions. The anteroposterior film can be dropped as a routine part of the screening radiographic evaluation for patients with possible cervical spine trauma. By not routinely obtaining this view, efficiency of trauma patient care is improved, less radiation is received by the patient, and health care costs are reduced. 


\title{
Comparison of Labetol, Diazepam and Haloperidol for the treatment of cocaine toxicity in a swine model
}

\author{
W. H. SPIVEY, J. M. SCHOFFSTALL, \\ R. KIRKPATRICK \& L. FUHS \\ Department of Emergency Medicine, Division of Research, The Medical College of \\ Pennsylvania, Philadelphia, U.S.A.
}

Study hypothesis. Drugs that inhibit seizure activity will diminish the toxic effects of an acute cocaine overdose. This study compares diazepam (D), labetalol (L), and haloperidol $(\mathrm{H})$ for the control of seizures and hypertension in an acutely cocaine toxic swine model.

Study population. Nineteen unanaesthetized swine $(10-15 \mathrm{~kg})$ with chronically implanted arterial and venous catheters.

Methods. All animals received a cocaine infusion of $1 \mathrm{mg} / \mathrm{kg} / \mathrm{min}$ IV with continuous monitoring of blood pressure (BP) and heart rate (HR). After $10 \mathrm{~min}$, the animals received D $0.2 \mathrm{mg} / \mathrm{kg}(\mathrm{n}=4), \mathrm{L} 1.5 \mathrm{mg} / \mathrm{kg}(\mathrm{n}=5)$, and $\mathrm{H} 0.1 \mathrm{mg} / \mathrm{kg}(\mathrm{n}=5) \mathrm{IV}$ over $1 \mathrm{~min}-5$ animals served as controls (C). Cocaine infusion was continued until the animals developed ventricular arrhythmias, status epilepticus or respiratory compromise. Blood was drawn every $5 \mathrm{~min}$ for measurement of epinephrine and norepinephrine levels. Data were analysed by ANOVA with Tukey A post-hoc tests.

Results. BP and HR increased in all animals with the infusion of cocaine. With the infusion of $\mathrm{D}, \mathrm{L}$, or $\mathrm{H}$, there was no significant change in either parameter at 5 min. All animals in the $C, L$, and $H$ groups developed multiple seizures after a mean \pm SD of $14.8,18.2$ and 15.7 min respectively. Only 2 of 4 animals developed seizures in the $D$ group, mean $-35 \mathrm{~min}(P<0.05)$. The cocaine infusion was stopped because of toxicity at a mean \pm SD of $20 \pm 2,41 \pm 8,21 \pm 3$, and $31 \pm 10 \mathrm{~min}$ for the $\mathrm{C}, \mathrm{D}, \mathrm{L}$ and $\mathrm{H}$ groups respectively. There was no significant difference for epinephrine or norepinephrine among the four groups.

Conclusion. Multiple seizures are a significant factor in the pathogenesis of cocaine toxicity. Diazepam offers the greatest protection for cocaine-induced seizures and toxicity. 


\section{Evaluation of the febrile adult in the emergency department}

\section{J. S. STRAPCZYNSKI}

Presbyterian-University Hospital, Emergency Department, University of Pittsburgh, Center for Emergency Medicine, Pittsburgh, U.S.A.

Study hypothesis. There are definable clinical criteria useful in evaluating febrile? adults in the emergency department (ED) that will reduce the utilization of ancillary tests and bacteriologic cultures.

Study population. Adult patients seen in an urban university hospital ED over a 12-month period with a complaint of fever or oral temperature $>38.0 \mathrm{C}$.

Methods. Retrospective review of ED, hospital, and clinic charts.

Results. A total of 26173 adult patients seen in the ED with $7590(29 \%)$ ad -8 missions. A total of $1547(6 \%)$ patients met entry criteria; $759(49 \%)$ admitted aftes ED evaluation, $219(14 \%)$ directly admitted without full ED evaluation, $541(35 \%)$ discharged, and $28(2 \%)$ transferred. Admitted patients were older and more likely to have comorbid diseases $(\mathrm{P}<.05$ by $2 \times 2$ table) than discharged patients. Localized signs and symptoms led to ED diagnosis that agreed with dischargeo diagnosis in $92 \%$ of admitted patients. Bacteremia was seen only in patients with: $\overrightarrow{0}$ (1) predisposing conditions; (2) localized infections, or (3) advanced age. The white cell count (WBC) or differential had no utility in young and middle aged adults in detecting those patients with bacterial infection. In the febrile elderly patient, bacterial infections were associated with leukocytosis, pyruia, or radio $\exists$ graphic infiltrate.

Conclusions. Febrile young and middle aged adults presenting to the ED can beo evaluated using signs and symptoms to guide the use of ancillary tests. In elderly patients, urinalysis, chest radiography, and WBC are helpful. Blood cultures areo useful only in patients with definable risk factors for bacteremia. 


\section{An analysis of emergency physicians' cumulative career risk of $\mathrm{HIV}$ infection}

R. L. WEARS, L. L. FLUSKEY, S. LI \& D. J. VUKICH

Division of Emergency Medicine, Department of Surgery, University of Florida, Jacksonville, U.S.A.

Hypothesis. Although emergency physicians' risk of contracting human immunodeficiency virus (HIV) infection from any single patient encounter is small, the cumulative risk over many years of practice is suprisingly large.

Population. A simulation analysis consisting of 5000 instantiations of career risk scenarios.

Methods. The risk of contracting HIV infection was modeled as a function of the annual risk of parenteral occupational exposure to contaminated body fluids, the probability of transmission given exposure, and an estimated rate of increase in the prevalence of HIV-seropositivity in the patient population. The risk of exposure was modelled by a Poisson distribution (mean $=0.29$ exposures per year for the base year); the probability of transmission was modelled by a beta distribution (mean \pm standard deviation $0.005 \pm 0.004$ ), as was the annual proportional increase in prevalence $(0.05 \pm 0.04)$; this value was progressively reduced to zero over 15 years. Several thousand simulation runs were made, allowing estimation of the expected cumulative risk, and of the actual distribution of risk.

Results. The mean of the 30-year risk of HIV infection was almost $5 \%$, while the range ran from a low of $1.7 \%$ to a high of greater than $10 \%$. The magnitude of risk depended on the length of the emergency physicians' career; with several decades of exposure, the effect of anti-HIV precautions appeared to be diminished.

Conclusions. Over their professional lifetimes, emergency physicians may be at substantially greater risk than generally assumed. Although beneficial at all times, the efficacy of anti-HIV precautions diminished with increasing length of active practice. 


\section{A comparison of mechanical CPR and manual CPR by monitoring end-tidal $\mathrm{pCO}_{2}$ in human cardiac arrest}

K. R. WARD, J. J. MENEGAZZI \& R. R. ZELENAK

Affiliated Residency in Emergency Medicine, University of Pittsburgh, Center for Emergency Medicine of Western Pennsylvania, Pittsburgh and Uniontown Hospital, Uniontown, Pennsylvania, U.S.A.

Study hypothesis. Mechanical CPR produces greater cardiac outputs than manual CPR in humans, as demonstrated by end-tidal $\mathrm{pCO}_{2}\left(\mathrm{ETPCO}_{2}\right)$ values (which have been shown in both animals and humans to correlate with cardiac output during CPR).

Study population. Fifteen consecutive adults ranging in age from 33-78 yearo presenting to a university hospital in nontraumatic cardiac arrest were enrolle over 1 month.

Methods. This was a prospective randomized cross-over study. Protocols were begun after initial resuscitation attempts were unsuccessful. Patients received four alternating $5 \mathrm{~min}$ trials (two manual and two mechanical) being randomized to begin with either mechanical or manual CPR. Mechanical compressions were performed by a mechanical compressor (compression depth 2 inches). Both mechanical and manual compressions were delivered at a rate of $80 / \mathrm{min}$ with a fixed ventilatory volume delivered after every fifth compression. Persons performing 3 manual CPR were experienced AHA BLS providers, and no one performed manual CPR twice during the study. No $\mathrm{NaHCO}^{3}$ or epinephrine was administered. $\mathrm{ETPCO}_{2}$ was continuously monitored. Those performing manual compressions were blinded to the ETPCO 2 monitor. $\mathrm{ETPCO}_{2}$ values between techniques were compared using Tukey's test (significance of $\mathrm{P}<\cdot 05$ ).

Results. The mean $\mathrm{ETPCO}_{2}$ during mechanical $\mathrm{CPR}$ was $13.6 \mathrm{mmHg}(+/-4.15 \mathrm{sd})$ 을 compared to $6.9 \mathrm{mmHg}(+/-2.42)$ during manually performed CPR $(\mathrm{p}<0.001)$, a N difference of $97 \%$. Mechanical $\mathrm{ETPCO}_{2}$ was higher in all cases. No one was resuscitated.

Conclusion. This study suggests that cardiac output during mechanical CPR is significantly higher than during manual $\mathrm{CPR}$ as evidenced by significantly greater $\mathrm{ETPCO}_{2}$. 


\title{
This clinical and experimental investigations of the Stratbucker Chambers Effect on
}

\section{transthoracic impedence}

\author{
D. G. FERGUSON \& W. O'DOWD
}

Royal Hallamshire Hospital, Sheffield, U.K.

Stratbucker and Chambers noted a decrease in Transthoracic impedence with successive discharge across the thorax. In their document $3 \mathrm{M}$ Littman ${ }^{\mathrm{TM}}$ Brand Defib - PAD Technical Evaluation Project (Stratbucker \& Chambers, 1969) they stated that this phenomena was first described by them in 1969 and they refer to it's occurrence in dog experiments. It was subsequently described by them in 1972 (Chamber et al., 1972) and again referred to by Kerber in 1981.

We have investigated this phenomena in 10 cardiac arrest cases and also 10 Cadaver studies. The Cadaver studies were part of an ongoing research project into the feasibility of using Cadavers as an experimental model for certain aspect of defibrillation research. We were unable to confirm the existence of the Stratbucker Chambers Effect in either the cardiac arrest cases nor in the Cadaver studies.

On the basis that others had described the Stratbucker Chambers Effect it was considered that one explanation of the effect might have been an alteration in contact between the skin, the electrolyte cream and the paddles. Some of the commercial creams contained an abrasive. Five percent Alcian Blue was added to a commercial cream which contained an abrasive. Biopsies were taken from below both paddles after one and then five discharges at 200 Joules. Subsequent histology failed to reveal any gross change in the skin and there was no evidence of penetration of the dye into the skin.

A further consideration of the explanation of the Stratbucker Chambers Effect was the fact that their paper (Chamber et al., 1972) describes a defibrillator which utilized a trapezoidal wave form. The voltages were at 1200 and 600 volts. Our defibrillator had a damped sinusoidal wave form. To produce a 600 volt peak, it only required an 8 Joule setting and to produce a 1200 volt peak it required a 32 Joule setting. On the basis that the Stratbucker Chambers Effect might only be present at lower discharge energies we carried out further Cadaver studies at 10 and 20 Joule discharges. We were again unable to reproduce the Stratbucker Chambers Effect. 


\title{
REFERENCES
}

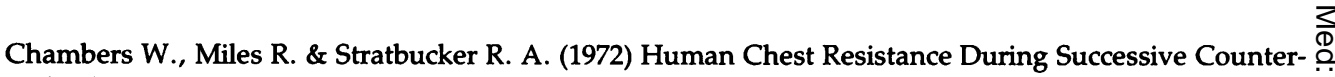
shocks. (ABSTR) Circulation 56 (Suppl 111) 111-183.

Kerber R. E., Grayzel J., Hoyt R., Marcus M. \& Kennedy J. (1981) Transthoracic Resistance In Human $\stackrel{\bar{\equiv}}{\stackrel{2}{2}}$ Defibrillation Circulation 63 (3), 676-682.

Stratbucker R. A. \& Chambers W. (1969) $3 M$ Littman $^{T M}$ Brand Defib-Pad Technical Evaluation Project.

\section{Conjunctival oxygen monitoring in the initial assessment of trauma}

\author{
J. HEYWORTH
}

Queen Alexandra Hospital, Portsmouth, U.K.

Recent studies have highlighted the number of preventable deaths from trauma, many of which are due to hypovolaemia diagnosed late thus allowing irreversible hypoxic injury to vital organs because of delayed transtusion and surgery. It is $\overrightarrow{0}$ becoming increasingly recognized that the standard parameters for detecting ear hypovolaemia are inaccurate and unreliable. Invasive monitoring is usually i appropriate during the early stages of assessment and resuscitation in the receiving A\&E department. The conjunctival oxygen sensor is a non-invasive monitor which, in animal (Smith \& Abraham, 1986) and human (Abraham et al., 1984) studies, has been shown to be an accurate index of early hypovolaemia.

Conjunctival oxygen tension was monitored in patients attending the A\&E 을 department of a district general hospital after trauma. The study sought (1) to detect hypovolaemia in those patients with normal standard physiological parameters and (2) to exclude hypovolaemia in, for example, the severely head injured $\triangle$ patient. Low measurements of conjunctival oxygen tension (less than $45 \mathrm{mmHg}$ ) shortly after arrival in the A\&E department were found in patients with hypovolaemia, although blood pressure and pulse were maintained, and reduced cardiac $\delta$ output. No patient with normal conjunctival oxygen tension was subsequently $₹$ found to be hypovolaemic.

The results of this study indicate that monitoring conjunctival oxygen tension provides a reliable adjunct in the early assessment of victims of trauma.

\section{REFERENCES}

Abraham E., Oye R., \& Smith M. (1984) Detection of blood volume deficit through conjunctival oxygen $\stackrel{0}{\Phi}$ tension monitoring. Critical Care Medicine, 12, 931-4. 
Smith M. \& Abraham E. (1986) Conjunctival Oxygen Tension Monitoring during haemorrhage. Journal of Trauma 26, 217-24.

\section{A mechanism for the modification of the cardiac response to haemorrhage by peripheral injury}

E. KIRKMAN, H. W. MARSHALL \& R. A. LITTLE

North Western Injury Research Centre, Stopford Building, University of Manchester, Manchester M13 9PT, U.K.

A progressive 'simple' haemorrhage, where there is little tissue damage, induces an initial tachycardia followed by a later bradycardia (Secher \& Bie, 1985; Little et al., 1989). The late bradycardia is reflex in nature (Little et al., 1989) due to the activation of cardiac-vagal afferent C-fibres (Oberg \& Thorén, 1972). This reflex which may be cardio-protective, leads to a marked fall in blood pressure (Little et al., 1989) and should be treated by re-expansion of the blood volume. However, when the haemorrhage is accompanied by tissue damage and nociception the bradycardia is absent (Little et al., 1989). The aim of the present study was to examine the mechanism of this effect of injury by determining whether nociceptive afferent activity could suppress the bradycardia induced by specific activation of cardiac-vagal afferent C-fibres.

Experiments were conducted on cats anaesthetized with intravenous 'Saffan' (12-20 mg.kg. ${ }^{-1} \mathrm{~h}^{-1}$ for surgery, $3-9 \mathrm{mg} . \mathrm{kg} .{ }^{-1} \mathrm{~h}^{-1}$ during tests, Glaxovet). In seven tests in four cats stimulation of cardiac afferent $\mathrm{C}$-fibres with phenylbiguanide $\left(16 \cdot 1-38.5 \mu \mathrm{g} . \mathrm{kg}^{-1}\right)$ injected into the left atrium produced a significant bradycardia, with heart rate falling by $92.0 \pm 9.8$ from $189.9 \pm 5.9$ beats. min $^{-1}$ (mean \pm sem, $\mathrm{P}<0.05$, Student's t-test), a marked significant fall in arterial blood pressure of $61.1<8.7 \mathrm{mmHg}$ from $132.0 \pm 5.2 \mathrm{mmHg}$ and a small reduction in respiratory minute volume of $0.011 \pm 0.002$ from $0.188 \pm 0.0441 . \mathrm{min} .^{-1} \mathrm{~kg}^{-1}$. Electrical stimulation on the central, cut end of the sciatic nerve $(10-20 \mathrm{~V}, 5 \mathrm{~ms}, 10 \mathrm{~Hz})$ to active somatic afferent $\mathrm{C}$-fibres, and hence mimic injury produced a significant increase in heart rate to $212.3 \pm 7.2$ beats. min. ${ }^{-1}$, blood pressure to $165.6 \pm 6.9 \mathrm{mmHg}$ and in respiratory minute volume to $0.255 \pm 0.0311 . \mathrm{min}^{-1} \cdot \mathrm{kg}^{-1}$. When phenylbiguanide was injected on a background of sciatic nerve stimulation its bradycardiac and hypotensive effects were significantly reduced with heart rate and blood pressure falling by only $26.9 \pm 6.5$ beats. $\mathrm{min}^{-1}$ and $7.6 \pm 2.9 \mathrm{mmHg}$ respectively, while the respiratory depression was abolished. A similar inhibition of the phenylbiguanide response by sciatic stimulation could also be seen when respiration was stopped 
transien

These results indicate that the depressor reflex elicited by activation of cardiac- $\frac{\bar{D}}{0}$ vagal afferent $C$-fibres may be inhibited by concomitant nociceptive afferent activity. This inhibition is unrelated to any nociception-induced respiratory changes $\frac{\text { f }}{0}$ and may explain the suppression by tissue injury of the bradycardia normally associated with a severe 'simple' haemorrhage.

\section{REFERENCES}

Little R. A., Marshall H. W. \& Kirkman E. (1989) Quarterly Journal of Experimental Physiology, 74, 825-33.

Oberg B. \& Thorén P. (1972) Acta Physiologica, Scandinavica, 85, 164-173.

Secher N. H. \& Bie P. (1985) Clinical Physiology, 5, 315-23.

\section{E.C.G. reporting in accident \& emergency: the minimum we should offer}

\section{N. J. FOTHERGILL \& S. M. MOALYPOUR}

Accident E Emergency Dept., Wolverton Avenue, Kingston-Upon-Thames, Surrey, U.K.

An E.C.G. which is thought to be normal often encourages a doctor to discharge $a^{\frac{\bar{\rho}}{3}}$ patient who presented with possible cardiovascular symptoms from the A\&E department.

Routine daily reporting of E.C.G.s of all patients discharged without hospital ${ }^{\mathrm{D}}$. follow up was carried out by a senior registrar in A\&E who had immediate access to the patient's records, to see whether significant E.C.G. abnormalities had been missed. The first 200 reports were assessed.

Just over half the patients had presented with chest pain, and $87 \%$ were seeno only by an A\&E doctor. Abnormalities were missed in 37 patients who were discharged home, and these were significant in 11 patients : four patients had을 been having an acute myocardial infarction, five their first presentation with angina, one myocarditis and one a bradyarrythmia which had led to a period of $\tilde{N}$ unconsciousness. Six of these significant errors were made by A\&E doctors and N్ల్ five by medical SHOs and Registrars. All patients were recalled or their G.P's contacted within $24 \mathrm{~h}$ and informed of the possible diagnosis; all survived. The commonest E.C.G. abnormality to be missed was abnormal $\mathrm{T}$ wave inversion, and the significance of conduction defects was not appreciated. 
Based on the findings of this study a simple protocol for reading E.C.G.s was introduced and taught to junior doctors in A\&E and in medicine. The effects of this teaching are being assessed.

The screening of E.C.G.s of patients discharged without hospital follow-up and the use of a teaching protocol such as ours is the very least that every major A\&E department should offer.

\section{Junior hospital doctors response to a megacode}

D. BARTON \& C. THOMAS

Leicester Royal Infirmary, Leicester LE1 5WW, U.K.

The resuscitation skills of junior hospital doctors has been described as inadequate (Casey, 1984; Kinner et al., 1985). The advent of the Report from the Royal College of Physicians on Resuscitation from Cardiopulmonary arrest Training and Organization has in part addressed the problem. Resuscitation training officers are being appointed with increasing frequency to teaching hospitals. The Resuscitation Council has laid down guidelines on the management of cardiopulmonary arrest. These guidelines may be tailored to the requirements of individual hospital resuscitation committees such as the choice of second line antiarrhythmic agents. In addition a number of Advanced Cardiac Life Support Courses are now available for those suitably motivated to increase or organize their skills. It has been recommended that medical schools place a greater emphasis on cardiopulmonary resuscitation and introduce an assessment thereof in final MB examinations. (Royal College of Physicians, 1987).

Junior Hospital Doctors were assessed at the beginning of their 6-month appointment in cardiopulmonary resuscitation skills. They were given a copy of the Resuscitation guidelines of the Resuscitation Committee of the Leicester Royal Infirmary. Each participant was informed that he or she would be assessed in resuscitation skills according to these guidelines within 2 weeks of commencing employment.

The assessment took the form of a megacode, using a Ventricular Fibrillation scenario. Their responses were recorded on a videotape. They $(\mathrm{N}=39)$ were assessed on 52 parameters of Basic and Advanced Cardiac Life Support. The results are discussed with reference to the Royal College of Physicians Report on Resuscitation from Cardiopulmonary Arrest Training and Organization (1987). If a standard existed in the UK such as those set by the American Heart Association's Advanced Cardiac Life Support Course (1988), less than half (46\%) of the candidates would have passed. 


\section{REFERENCES}

Casey W. F. (1984) Cardiopulmonary resuscitation: a survey of standards amongst junior hospital doctors. Journal of the Royal Society of Medicine, 77, 921-24.

Skinner D. V., Camm A. J., Miles S. (1985) Cardiopulmonary resuscitation skills of pre-registration house officers. British Medical Journal, 290, 1549-50.

Resuscitation from cardiopulmonary arrest. Training and organisation. (1987) Journal of the Royal College of Physicians, London, 21, 175-82.

Guidelines for cardiopulmonary resuscitation. Revised recommendations of the Resuscitation Council (UK). (1989) British Medical Journal, 299, 442-8.

Instructors manual for Advanced Cardiac Life Support. American Heart Association (1988) Dallas.

\section{A review of the management of oral drug overdose in the accident and emergency department}

\section{W. HODGKINSON}

Accident and Emergency Department, Hope Hospital Salford, Manchester, U.K.

We present a study showing that oral or nasogastrically delivered activated charcoal can replace the use of syrup of ipecac and orogastric lavage in the routine management of oral drug overdose.

A prospective study was performed on 289 patients who made a total of 323 presentations to the Royal Brisbane Hospital Accident and Emergency department. All had a known or suspected oral drug overdose.

The treatment protocol removed the use of syrup of ipecac completely and reduced the need for orogastric lavage to a minimum ( $4 \%$ of patient presentations). A charcoal and sorbitol slurry was given to $61 \%$ of patient presentations. This did not adversely affect the morbidity or mortality $(0.3 \%)$ of these patients when compared with other studies. Furthermore, $76 \%$ of these patients could be managed in an Accident and Emergency observation unit.

We conclude that activated charcoal should replace syrup of ipecac and orogastric lavage in the routine management of these patients. 


\title{
Evidence for a central inhibition of heat loss during the acute phase of burn injury in children
}

\author{
C. CHILDS, H. B. STONER \& R. A. LITTLE
}

North Western Injury Research Centre, University of Manchester, U.K.

At past meetings of this society we presented data to show an early disturbance in thermoregulation after burn injury in young children. Deep body (rectal) temperature rises to reach a peak, $12 \mathrm{~h}$ after the accident and total body heat content increases significantly above control values. An increase in heat loss might be expected to offset the increased heat storage but our recent studies reveal that there is an active inhibition of heat loss in areas of the body concerned with thermoregulation.

Fourteen patients aged 5-53 months (median $10 \mathrm{~m}$ ) with $9-15 \%$ burns (median $12 \%$ ) and 16 healthy children aged 7-77 months (median $30 \mathrm{~m}$ ) were studied bandaged for $1-12 \mathrm{~h}$ (controls) and $24 \mathrm{~h}$ (patients) at $\mathrm{Ta} 30^{\circ} \mathrm{C}$. Heat loss by dry routes, radiation and convection, was calculated from measurements of body surface and ambient temperature and a heat exchange coefficient. Evaporative heat loss was calculated from measurements of evaporation rate (ER).

The high ER over the burn wound $\left(72.2 \pm 23.6 \mathrm{~g} \cdot \mathrm{m}^{2} \mathrm{~h}^{-1}\right)$ was significantly reduced by bandaging $\left(38.7 \pm 15.8 \mathrm{~g} \cdot \mathrm{m}^{2} \mathrm{~h}^{-} 1\right)$. These rates of ER represent an uncontrolled loss of water (and heat). ER measured over the unburned skin (which is still under central nervous control) was significantly lower than at the same sites in controls. The reduced ER over normak skin in the patients occurred at Ta $30^{\circ} \mathrm{C}$ and in the presence of a marked increase in both core $\left(38.9 \pm 0.6^{\circ} \mathrm{C}\right.$, mean \pm S.D. $)$ and skin $\left(37.0 \pm 0.6^{\circ} \mathrm{C}\right)$ temperature.

Heat loss by the dry routes was significantly higher in the patients than controls. Radiant heat loss in patients was $29.0 \pm 1.2 \mathrm{Wm} 2$ (mean \pm sem) and in controls, $22.3 \pm 2.0 \mathrm{Wm} 2(\mathrm{P}<0.05$, Wilcoxon rank sum test). Convective heat loss in patients was $14.4 \pm 0.6 \mathrm{Wm} 2$ and in controls, $9.7 \pm 0.7 \mathrm{Wm} 2(\mathrm{P}<0.001)$. The greater rates of heat loss by the dry routes can be explained by the higher temperature gradient between the body surface and the environment. Although the increased skin temperatures provided a vehicle for increased dry heat loss over a large area of the body surface there were areas which are normally involved in thermoregulation which were cooler. During the first $24 \mathrm{~h}$ post burn period peri- 
pheral vasoconstriction, with toe temperatures of $31.3 \pm 1.2^{\circ} \mathrm{C}$ and an increased core - peripheral temperature gradient, $7.6 \pm 1.6^{\circ} \mathrm{C}$, was a characteristic of thesez patients despite being adequately resuscitated and nursed in a warm $\left(30^{\circ} \mathrm{C}\right)^{\circ}$. environment. Under similar conditions the toe temperatures of apyrexial controls $\vec{*}$ was $34.4 \pm 0.9^{\circ} \mathrm{C}$ (mean \pm S.D.) with a core - peripheral temperature gradient of $2 \cdot 4 \pm 1 \cdot 0^{\circ} \mathrm{C}$.

These results show that the normal responses to an increased heat storage $; \frac{\bar{p}}{\vec{p}}$ sweating and peripheral vasodilatation, are inhibited soon after burn injuryळ in children. These changes are consistent with an upward movement of theis hypothalamic set-point temperature. We have evidence to support the activity of 0 cytokines as mediators of these changes.

\section{The variations in heart Rate and S
with blood loss following trauma}

\section{P. A. DRISCOLL}

University Department of Accident and Emergency Medicine, Hope Hospital, Salford, Manchester, U.K.

Experiments have shown that the Shock Index (S.I.) progressively rises as the magnitude of the blood loss increases. In contrast, the heart rate response to haemorrhage is biphasic. the concomitant tissue injury associated with traumat makes it difficult to determine the transition point from tachycardia to relative ${ }^{3}$ bradycardia because of the nociceptive afferent impulses and the limitations in estimating blood loss. An investigation was therefore carried out on a selected. group of patients with low levels of tissue damage and in whom the degree o® haemorrhage could be measured. The alteration in heart rate and S.I., with bloods loss, was recorded.

This prospective study was carried out on adult, male victims of stab wounds़ who attended the Emergency Departments of either Johannesburg General Hospital or Baragwanath Hospital. Factors which are known to affect heart rate were recorded. In 40 cases the following criteria applied. The patients had not beer? pharmacologically paralysed and had not received more than 1 litre of crystalloid丸 during the pre-hospital phase. There was no history of exposure or profuse external bleeding. Any associated head or spinal injury had an AIS level of less than 3. In all cases the blood loss was calculated from the amount of blood foun of in the abdomen and thorax at operation or released into containers after 15 min following insertion of a chest drain.

The heart rate was found to rise up to a plateau with blood losses between 1- $2^{2}$ 
litres (approximately $20-40 \%$ of the blood volume). Further bleeding produced a progressive fall in the heart rate. In contrast, the S.I. rose from 0.70 to 1.60 as the blood loss increased from $10-80 \%$.

These results show that the transition from tachycardia to bradycardia begins to occur with a blood volume loss of approximately $40 \%$. The findings also indicate that there was no binominal distribution in the S.I. with blood loss. This supports the concept of using the S.I. as an indicator of blood loss in a selected group of trauma patients.

\section{EMRS PRIZE}

\section{Investigation of a reversal agent for acute ethanol poisoning in an accident and emergency department}

\section{S. MARDEL, I. PHAIR \& J. A. HENRY}

Aberdeen Royal Infirmary, Aberdeen, Leicester Royal Infirmary, Leicester and National Poisons Information Service, U.K.

Intravenous pyridoxine was evaluated as an agent for the reversal of ethanolinduced central nervous depression in a randomized double-blind controlled study of 60 patients presenting to an accident and emergency department with a clinical diagnosis of acute ethanol intoxication. Level of consciousness, measured by a modified Glasgow coma scale, was significantly improved at $30 \mathrm{~min}$ after a single dose of pyridoxine. Mean time spent in the department was shortened in the pyridoxine treated group.

The patients studied had a mean blood ethanol concentration of $259 \mathrm{mg} / \mathrm{dl}$. The management of this difficult group of patients is discussed. 


\title{
EMRS PRIZE
}

\section{Plasma catecholamine responses in acute severe asthma}

\author{
T. PARKE, D. J. STEEDMAN C. E. ROBERTSON, \\ P. F. MAYCOCK \& R. A. LITTLE \\ Department of Emergency Medicine, Royal Infirmary of Edinburgh, North West Injuries \\ Research Centre, Manchester, U.K.
}

Study hypothesis. Acute severe asthma in man is associated with impaired catecholamine responses.

Study population. Twenty patients aged 19 to 71 years (mean 45 years) presenting to the Emergency Department with acute severe asthma were studied. The diagnosio was established on the basis of a history of reversible airflow obstruction together. with a reduction in peak expiratory flow rate of more than $65 \%$ from predicte values.

Methods. Prior to therapy, an IV cannula was sited and a $5 \mathrm{ml}$ sample was drawn into a heparinized tube on ice. Plasma was separated within $10 \mathrm{~min}$ and stored at $-80^{\circ} \mathrm{C}$ until analysis. Arterial blood gases were simultaneously sampled and pre and post treatment peak expiratory flow rates, pulse rate, the degree of pulsus paradoxus and respiratory rate were noted. Plasma catecholamine concentrations were measured by high performance liquid chromatography with electrochemical detection after preparation on cation exchange resin and alumina. Control values following venepuncture are $2.1 \pm 0 \cdot 2$ (mean $\pm \mathrm{SE}$ ) $\mathrm{nmol} / \mathrm{l}$ for noradrenaline (N.A.) and $0.39 \pm 0.07 \mathrm{nmol} / 1$ for adrenaline (A.).

Results. There was a significant correlation between the log plasma adrenaline levels and $\mathrm{PaCO}_{2}(\mathrm{P}=0.002)$, pulse rate $(\mathrm{P}=0.006)$, the reduction in peak flow $(P=0.012)$ and the degree of pulsus paradoxus $(P=0.049)$. There was no significant correlation between the log plasma adrenaline and $\mathrm{PaO}_{2}$ arterial oxygen concentration or respiratory rate. There was significant correlation between the log plasma of N.A. and $\mathrm{PaCo}_{2}(\mathrm{P}=0.032)$ and pulsus paradoxus $(\mathrm{P}=0.036)$ but no correlation with pulse rate, reduction in peak expiratory flow rate, $\mathrm{PaO}_{2}$ or respiratory rate. All patients made an uncomplicated recovery.

Discussion. Previous researchers have suggested an impaired catecholamine response in patients with acute severe asthma. Adrenaline is the most potent endogenous bronchodilator and it would be surprising if levels did not increase in 
a similar way to other acute conditions such as myocardial infarction, trauma and sepsis. The relationships demonstrated in this study between adrenaline, noradrenaline and the other parameters imply that only in life-threatening cases of asthma is the catecholamines response similar to that seen in these other states. The response to impending respiratory arrest appears to involve a surge of adrenaline release. Hypoxia does not appear to be the trigger for this but rather the development of hypercapnoea.

\section{EMRS PRIZE}

\section{Why does the milch manoeuvre work? an anatomical study}

\section{G. JOHNSON \& M. CLANCY}

Accident and Emergency Department, Pinderfields General Hospital, Wakefield, U.K.

The Milch manoeuvre has been shown to be an effective and atraumatic method for reducing anteriorly dislocated shoulders. In comparison with other more established techniques, the patient experiences less pain and a minimum amount of force is required to achieve reduction. Milch proposed that the success of the technique was due to the parallel orientation of the muscles acting on the shoulder in the abducted position. This conclusion was reached by drawing straight lines between the origin and insertion of the shoulder muscles onto radiographs of shoulders in neutral and abducted positions with the joint reduced. No attempt was made to study the dislocated joint.

We investigated the mechanism of reduction in the Milch manoeuvre by dissection of a cadaver shoulder and by development of an articulated model. It was also possible to compare the role of the muscles acting on the shoulder in the Milch and Kocher techniques.

We found that during abduction of the dislocated shoulder in the Milch technique, many of the major muscles around the shoulder were shortened, in particular subscapularis was shortened and its pull became oblique as it rode up over the humeral head. In the Kocher manoeuvre, the effect of subscapularis was directly opposed by stretching the muscle by forcible external rotation. These statements are supported by slides illustrating our dissections.

In conclusion, the Milch manoeuvre decreases the efficiency of subscapularis allowing reduction to occur with less force and less pain than with the Kocher technique. 


\title{
EMRS PRIZE
}

\section{Cardiac and oxygen transport responses to human serum albumin and gelatin} resuscitation of porcine haemorrhagic shock

\author{
M. Y. RADY, R. A. LITTLE, E. KIRKMAN \& \\ S. FAITHFUL* \\ North Western Injury Research Centre, Stopford Building, Manchester and *Delta \\ Biotechnology, Nottingham, U.K.
}

Colloid fluid therapy of hypovolaemic shock restores cardiac function and systemgico oxygen transport $\left(\mathrm{O}_{2} \mathrm{~T}\right)$ to optimal values with least risk of volume overlos $\mathrm{d}^{\circ}$

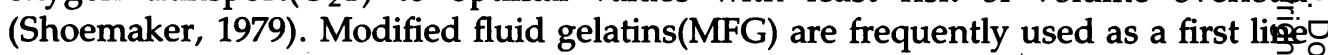
colloid fluid for expanding plasma volume because of their low cost and availability. We have examined cardiac and $\mathrm{O}_{2} \mathrm{~T}$ responses to resuscitation of haemorrhagico shock with either human serum albumin(HSA) or MFG. The study was conducted on anaesthetized immature domestic swine, weight $16-27 \mathrm{~kg}$ (7 animals in each group). General anaesthesia was induced with ketamine and maintained with $\mathrm{O}_{2}, \frac{0}{3}$ $\mathrm{N}_{2} \mathrm{O}$ and isoflurane up to $1.5 \%$. Instrumentation included bilateral femoral arterial cannulae (arterial pressure monitoring and bleeding) and a $7 \mathrm{~F}$ thermodilution pulmonary artery catheter (inserted via the right femoral vein). Animals were bled@ to a total of $30 \mathrm{ml} / \mathrm{kg}$ at a rate of $0.75 \mathrm{ml} / \mathrm{min} . \mathrm{kg}$. After $30 \mathrm{~min}$ colloids were infused:at $2 \mathrm{ml} / \mathrm{min} . \mathrm{kg}$ to a maximal volume of $45 \mathrm{ml} / \mathrm{kg}$. Cardiac and $\mathrm{O}_{2} \mathrm{~T}$ variables were measured before haemorrhage (control), end of haemorrhage(EH), end of re-o suscitation(ER) and 60 and $120 \mathrm{~min}$ later. $\mathrm{DO}_{2} \mathrm{I}$ was partially restored with colloids $₹$ although HSA seemed to have a longer lasting effect. Both colloids provided? adequate tissue oxygenation to correct the lactic acidosis and $\mathrm{O}_{2}$ debt sustained during shock. HSA and MFG augmented cardiac function to supranormal values. The increase in cardiac function induced by MFG was less well maintained than with HSA. The longer duration of the response to HSA, which may be due to its 0 greater intravascular half life, suggests that it is a better choice than MFG during the resuscitation of hypovolaemic shock. 


\title{
REFERENCE
}

Shoemaker W. C. (1979) Comparison of the relative effectiveness of whole blood transfusions and various types of fluid therapy in resuscitation. Critical Care Medicine, 4, 71-8.

\section{EMRS PRIZE}

\section{A study to determine the maximum tolerable dead space for the design of protective smokehoods}

\author{
P. D. MARTIN, J. A. S. ROSS \& S. J. WATT \\ Departments of Environmental and Occupational Medicine and Anaesthetics and \\ Intensive Care, Aberdeen Royal Infirmary, U.K.
}

Following the Manchester Air Disaster of 1985 and other incidents since, where individuals died following inhalation of smoke incapacitating them, to prevent an effective escape, attention has been paid to the development of apparatus to protect against the inhalation of smoke and its toxic constituents.

The Civil Aviation Authority (CAA) has determined the design specifications and the conditions in which any apparatus would be required to be worn. With specific levels of inspired oxygen, carbon dioxide and carbon monoxide defined as well as exercise levels and time course.

Proposed designs of smokehoods include a filter for the removal of toxins and irritants and the conversion of $\mathrm{CO}$ to $\mathrm{CO}_{2}$. There is no fresh gas supply and a dead space with respiratory implications is a necessity of their construction. As a result the ambient gases inspired are made further hypoxic and hypercarbic.

The aim of this study has been the definition of the maximum tolerable dead space for a wide variety of individuals (children to elderly) enduring the CAA determined scenario.

We have used a commercially available computer program that simulates cardiorespiratory physiology to define individuals and their response to the CAA conditions with added dead spaces of varying volumes.

The program was first scrutinized to determine its basis in the scientific literature. Next it was validated against data from healthy volunteers who were exposed to the CAA conditions in a laboratory. After this, modelling of 8-year-olds, 30-yearolds and 65-year-olds undergoing the experiment with a variety of addd dead spaces $(5 \% 15 \% 25 \%$ and $50 \%$ of their vital capacities) was performed. Finally the respiratory performance under these conditions was interpreted and recommendations for the maximum allowable dead space made. 